\title{
FIRST AND SECOND ORDER OPTIMALITY CONDITIONS FOR THE CONTROL OF FOKKER-PLANCK EQUATIONS*
}

\author{
M. SoledAd AronnA ${ }^{1, * *}$ And Fredi TrÖltzsch ${ }^{2}$
}

\begin{abstract}
In this article we study an optimal control problem subject to the Fokker-Planck equation

$$
\partial_{t} \rho-\nu \Delta \rho-\operatorname{div}(\rho B[u])=0
$$

The control variable $u$ is time-dependent and possibly multidimensional, and the function $B$ depends on the space variable and the control. The cost functional is of tracking type and includes a quadratic regularization term on the control. For this problem, we prove existence of optimal controls and first order necessary conditions. Main emphasis is placed on second order necessary and sufficient conditions.
\end{abstract}

Mathematics Subject Classification. 49J20, 49K20, 49K27, 35Q84.

Received February 10, 2020. Accepted February 2, 2021.

\section{INTRODUCTION}

In this article we prove first and second order optimality conditions for a control problem subject to the Fokker-Planck equation

$$
\partial_{t} \rho-\nu \Delta \rho-\operatorname{div}(\rho B[u])=0
$$

where $u$ is a time-dependent possibly multidimensional control, and $B[u](x)=c(x)+b(x) \otimes u$ is defined by given vector functions $c$ and $b$.

Fokker-Planck equations arise in many situations in which a large number of agents is involved. More precisely, these equations are known to describe the time evolution of the probability density function of agents, where the motion of each of them is modelled by a stochastic differential equation. In particular, Fokker-Planck equations are present in models of mass opinion dynamics [2], tumor growth [12, 27], bird flocks movement [21] and various biological events $[18,36]$, among others. The recent survey [24] describes several applications of the Fokker-Planck equation to different socio-economic phenomena. It is worth mentioning that some of these articles already analyze control problems associated to their models, as e.g. [2, 27].

\footnotetext{
* The first author was supported by CAPES (Brazil) and by the Alexander von Humboldt Foundation (Germany).

Keywords and phrases: optimal control, Fokker-Planck equation, existence of optimal control, first order optimality conditions, second order optimality conditions.

1 Escola de Matemática Aplicada, Fundação Getúlio Vargas, Praia de Botafogo 190, Rio de Janeiro 22250-900, Brazil.

${ }^{2}$ Institut für Mathematik, Technische Universität Berlin, Straße des 17. Juni 136, 10623 Berlin, Germany.

** Corresponding author: soledad.aronna@fgv.br
} 
Another motivation for investigating optimal control problems governed by Fokker-Plack equations comes from Mean Field Games (MFG) theory [26, 31]. It has been observed ([31], Sect. 4) (see also [15, 35]) that, under a specific choice of the objective functional, the MFG system can be interpreted as a first order optimality system for the control of a Fokker-Planck equation.

For a review on Fokker-Planck control frameworks, we refer the reader to the survey [6] and to the references therein. Optimal control problems governed by (1.1) have been recently studied in quite a number of articles. For numerical control strategies, we mention in particular $[4,5]$, where piecewise constant one-dimensional and multidimensional constant controls, respectively, are discussed. The case of space-dependent time-independent control is investigated in [34]. Moreover, we refer to the following papers that are closer to our control problem and mainly concentrate on aspects of the analysis. For a more general setting with time- and space-dependent control, existence results and first order optimality conditions are proved in [1,23]. Their results on first order analysis are similar to ours. We also mention [13], where the authors show results on stabilization of (1.1) through linearization (more details in Rem. 2.1).

Optimization problems associated to (1.1) belong to the class of bilinear optimal control. This framework has been considered in e.g. [17] for elliptic equations. On the other hand, the work [7] dealt with infinite dimensional bilinear dynamical systems, but their results do not apply here.

In this paper we provide first and second order optimality conditions for an optimal control problem associated to (1.1), under quite mild regularity assumptions on the data functions and the spatial domain of the state equation. The cost functional is of tracking type and includes a quadratic regularizing term on the control. The control is only time-dependent. We obtain our second order necessary and sufficient conditions by application of results proved in [16] in an abstract framework.

Our paper is organized as follows. In Section 2, we present the equation, the basic assumptions, show wellposedness and some other properties of the state equation. The optimal control problem is introduced in Section 3, where existence of optimal solution is proved. In Section 4, we discuss properties of the controlto-state mapping, while Section 5 presents the adjoint system and first order optimality conditions. Section 6 is devoted to second order analysis and contains our main theorems. Finally, in the Appendix A we included some proofs of auxiliary results.

\subsection{Notation}

Given a real interval $[0, T]$, a normed space $X$ and $p \in[1, \infty]$, we let $L^{p}(0, T ; X)$ denote the Lebesgue space of $L^{p}$-functions and write $C([0, T] ; X)$ for the space of continuous functions, both with domain $[0, T]$ and values in $X$. When $X=\mathbb{R}$ we just write $L^{p}(0, T)$ and, for any $m$, we let $\|\cdot\|_{p}$ denote the norm in $L^{p}\left(0, T ; \mathbb{R}^{m}\right)$. Analogously, for a set $\Omega \subseteq \mathbb{R}^{n}, L^{p}\left(\Omega ; \mathbb{R}^{m}\right)$ is the Lebesgue space of $L^{p}$-functions with domain $\Omega$ and values in $\mathbb{R}^{m}$. We omit $\mathbb{R}^{m}$ when $m=1$ and the values range in $\mathbb{R}$, and we use the form $\|\cdot\|_{L^{p}(\Omega)^{m}}$ to denote the norm in $L^{p}\left(\Omega, \mathbb{R}^{m}\right)$.

Throughout the article, we consider the real Hilbert spaces $L^{2}(\Omega), H^{1}(\Omega)$ and $H^{1}(\Omega)^{*}$. We let $(\cdot, \cdot)$ denote the scalar product in $L^{2}(\Omega)$ and $\langle\cdot, \cdot\rangle$ the dual pairing between $H^{1}(\Omega)^{*}$ and $H^{1}(\Omega)$. Other scalar products and pairings will be distinguished by specifying the spaces as subindexes. For two vectors $u, v \in \mathbb{R}^{n}$, the result of the componentwise multiplication $u \otimes v$ is defined by the vector $w \in \mathbb{R}^{n}$ with components $w_{i}=u_{i} v_{i}$, for $i=1, \ldots, n$.

\section{The COntrolled FokKer-Planck EQUation}

We consider the Fokker-Planck equation with initial and boundary conditions given by

$$
\begin{aligned}
\partial_{t} \rho(x, t)-\nu \Delta \rho(x, t)-\operatorname{div}(\rho(x, t) B[u(t)](x)) & =0 \quad \text { in } Q, \\
\rho(x, 0) & =\rho_{0}(x) \quad \text { in } \Omega, \\
(\nu \nabla \rho(x, t)+\rho(x, t) B[u(t)](x)) \cdot n(x) & =0 \quad \text { on } \Sigma,
\end{aligned}
$$


where $\nu>0, \rho_{0} \in L^{2}(\Omega), \Omega \subset \mathbb{R}^{n}$ is a bounded domain with Lipschitz boundary $\Gamma:=\partial \Omega$, and we set $\Sigma:=$ $\Gamma \times(0, T), Q:=\Omega \times(0, T)$. The control is $u=\left(u_{1}, \ldots, u_{n}\right) \in L^{\infty}\left(0, T ; \mathbb{R}^{n}\right)$, and the function $B: \mathbb{R}^{n} \times \Omega \rightarrow \mathbb{R}^{n}$ is given by

$$
B[u](x):=c(x)+b(x) \otimes u,
$$

where $c, b \in L^{\infty}\left(\Omega ; \mathbb{R}^{n}\right)$ are fixed. In (2.1), the differential operators $\Delta$ and div act only with respect to the spatial coordinate $x$. For definition and basic properties of the div-operator we refer to [25]; in particular, we frequently use the Green's formula ([25], (2.17), p. 28). In (2.3), $n$ denotes the outward normal unit vector on $\Gamma$.

Remark 2.1. Let us compare this equation with others considered in the literature.

Breiten, Kunisch and Pfeiffer [13] assume $B$ to take the form $\nabla_{x} V$ for a potential $V$ given by

$$
V(x, t)=G(x)+\gamma(x) u(t)
$$

for a scalar control $u$ and study the infinite horizon stabilization problem. In [14], the same authors investigate the value function associated to a general class of infinite horizon optimal control problems that includes the control of Fokker-Planck equations.

Fleig and Guglielmi [23] consider a distributed multidimensional control and adopt an homogeneous Dirichlet boundary condition. In that case, the measure $\int_{\Omega} \rho(t, x) \mathrm{d} x$ is not preserved. For that problem, they show existence of optimal control and first order optimality conditions. Similar results, for distributed control and for a non-flux boundary condition as ours, were obtained by Albi et al. [1].

Equation (2.1), for a function $u$ that depends both on time and space, appears in second order Mean Field Games: see e.g. Gomes and Saúde ([26], Thm. 2), Lasry and Lions ([31], Sect. 2.6).

\subsection{Existence and uniqueness of the solution of the Fokker-Planck equation}

We start by obtaining the weak formulation of (2.1)-(2.3) by standard calculations. In particular, we apply the formula

$$
-\int_{\Omega} \operatorname{div}(\rho B[u]) \varphi \mathrm{d} x=-\int_{\Gamma} \varphi \rho B[u] \cdot n \mathrm{~d} s+\int_{\Omega} \rho B[u] \cdot \nabla \varphi \mathrm{d} x
$$

that will frequently be needed. Multiplying (2.1) by a sufficiently smooth $\varphi$, integrating by parts, and using the boundary condition (2.3), we get

$$
\int_{\Omega} \partial_{t} \rho \varphi \mathrm{d} x+a[u(t)](\rho, \varphi)=0
$$

where, for each $u \in \mathbb{R}^{n}, a[u](\cdot, \cdot)$ is a bilinear mapping that to each pair $\psi, \varphi \in H^{1}(\Omega)$ associates the value

$$
a[u](\psi, \varphi):=\int_{\Omega}(\nu \nabla \psi+\psi B[u]) \cdot \nabla \varphi \mathrm{d} x
$$

We will work in the space

$$
W(0, T):=\left\{\rho \in L^{2}\left(0, T ; H^{1}(\Omega)\right): \partial_{t} \rho \in L^{2}\left(0, T ; H^{1}(\Omega)^{*}\right)\right\}
$$


equipped with the norm

$$
\|\rho\|_{W(0, T)}:=\left(\|\rho\|_{L^{2}\left(0, T ; H^{1}(\Omega)\right)}^{2}+\left\|\partial_{t} \rho\right\|_{L^{2}\left(0, T ; H^{1}(\Omega)^{*}\right)}^{2}\right)^{1 / 2}
$$

It is known that $W(0, T)$ is a Hilbert space with the scalar product

$$
(\rho, \varphi)_{W(0, T)}:=\int_{0}^{T}(\rho(t), \varphi(t))_{H^{1}(\Omega)} \mathrm{d} t+\int_{0}^{T}\left(\partial_{t} \rho(t), \partial_{t} \varphi(t)\right)_{H^{1}(\Omega)^{*}} \mathrm{~d} t
$$

which induces the norm $\|\cdot\|_{W(0, T)}$. We shall also recall the following continuous embedding (see e.g. Chipot [19], Thm. 11.4 or Dautray-Lions [20], Thm. 1, p. 473)

$$
W(0, T) \hookrightarrow C\left([0, T] ; L^{2}(\Omega)\right)
$$

that will be of use throughout this article.

The weak formulation of (2.1) can be rewritten as to find $\rho \in W(0, T)$ such that

$$
\begin{aligned}
\frac{\mathrm{d}}{\mathrm{d} t}(\rho(\cdot), \varphi)+a[u(\cdot)](\rho(\cdot), \varphi) & =0 \quad \text { on } \mathcal{D}^{\prime}(0, T) \text { for all } \varphi \in H^{1}(\Omega), \\
\rho(0) & =\rho_{0} \quad \text { in } L^{2}(\Omega) .
\end{aligned}
$$

For convenience, let us consider a general right-hand side $f \in L^{2}\left(0, T ; H^{1}(\Omega)^{*}\right)$ in $(2.6)$ and study the existence and uniqueness of the solution of the equation

$$
\frac{\mathrm{d}}{\mathrm{d} t}(\rho(\cdot), \varphi)+a[u(\cdot)](\rho(\cdot), \varphi)=\langle f(\cdot), \varphi\rangle \quad \text { on } \mathcal{D}^{\prime}(0, T) \text { for all } \varphi \in H^{1}(\Omega)
$$

with initial condition (2.7).

Remark 2.2. Note that (2.8) can also be expressed as

$$
\partial_{t} \rho+a[u](\rho, \cdot)=f \quad \text { in } L^{2}\left(0, T ; H^{1}(\Omega)^{*}\right) .
$$

Definition 2.3. We call a function $\rho \in W(0, T)$ a weak solution of the Fokker-Planck equation (2.1), if it satisfies (2.6)-(2.7). The definition is formulated analogously for (2.8)-(2.7), where the r.h.s. of the differential equation in (2.1) was replaced by an arbitrary element $f$ of the space $L^{2}\left(0, T ; H^{1}(\Omega)^{*}\right)$.

Remark 2.4. For practical matters, we mention the equivalent variational formulation of (2.1)-(2.3) according to Ladyzhenskaya et al. [30] that is as follows: $\rho$ is a function in $W_{2}^{1,0}(Q)$ such that

$$
\iint_{Q}\left\{-\rho \partial_{t} \varphi+(\nu \nabla \rho+\rho B[u]) \cdot \nabla \varphi\right\} \mathrm{d} x \mathrm{~d} t=\int_{\Omega} \rho_{0} \varphi(\cdot, 0) \mathrm{d} x, \quad \text { for all } \varphi \in W_{2}^{1,1}(Q) \text { with } \varphi(\cdot, T)=0 .
$$

Here, $W_{2}^{1,0}(Q)$ is the Banach space of all $\rho \in L^{2}(Q)$ that have the weak derivatives $\partial_{x_{i}} \rho$ in $L^{2}(Q)$ for all $i \in\{1, \ldots, n\}$ and $W_{2}^{1,1}(Q)$ is the subspace of all $\rho \in W_{2}^{1,0}(Q)$ that also possess the derivative $\partial_{t} \rho$ in $L^{2}(Q)$. We refer to [30] for the definition and the associated norms.

Existence and uniqueness of a weak solution to (2.1)-(2.3) was proved by Breiten et al. [13] for controls $u \in L^{2}(0, T)$. The result was also given by Albi et al. [1] for the more general control space $L^{2}\left(0, T ; L^{\infty}(\Omega)\right)$. Both articles assume smoothness of the domain's boundary. Here we work on a Lipschitz domain and with controls 
in $L^{2}\left(0, T ; \mathbb{R}^{n}\right)$. This extension to $L^{2}\left(0, T ; \mathbb{R}^{n}\right)$ of the existence and uniqueness result for the weak solution of (2.1)-(2.3) is useful for the second order analysis we present later on. Hence, we prove the result again, but in a different way. We begin with bounded controls and then extend the result to controls in $L^{2}\left(0, T ; \mathbb{R}^{n}\right)$ by a density argument that is based on a priori estimates, as done in [13].

Theorem 2.5. Given $\rho_{0} \in L^{2}(\Omega), u \in L^{\infty}\left(0, T ; \mathbb{R}^{n}\right)$, and $f \in L^{2}\left(0, T ; H^{1}(\Omega)^{*}\right)$, there exists a unique weak solution $\rho$ of (2.8)-(2.7) and it belongs to the space $W(0, T)$.

Proof. We apply Lions-Magenes ([32], Thm. 4.1 and Rem. 4.3, pp. 238-239); see also Chipot ([19], Thm. 11.7).

We first verify the assumptions of continuity and coercivity of the bilinear form $a[u]$ necessary to apply ([32], Thm. 4.1 and Rem. 4.3, pp. 238-239). For $\rho, \varphi \in H^{1}(\Omega)$, we have

$$
\begin{aligned}
|a[u(t)](\rho, \varphi)| & \leq\left|\nu \int_{\Omega} \nabla \rho \cdot \nabla \varphi \mathrm{d} x\right|+\left|\int_{\Omega} \rho B[u(t)] \cdot \nabla \varphi \mathrm{d} x\right| \\
& \leq \nu\|\nabla \rho\|_{L^{2}(\Omega)^{n}}\|\nabla \varphi\|_{L^{2}(\Omega)^{n}}+\|B[u]\|_{L^{\infty}(Q)^{n}}\|\rho\|_{L^{2}(\Omega)}\|\nabla \varphi\|_{L^{2}(\Omega)^{n}} \leq C\|\rho\|_{H^{1}(\Omega)}\|\varphi\|_{H^{1}(\Omega)},
\end{aligned}
$$

where $C:=\nu+\|B[u]\|_{L^{\infty}(Q)^{n}}$. From the latter estimate and Young's inequality, we also obtain, for any positive $M$,

$$
\left|\int_{\Omega} \rho B[u] \cdot \nabla \varphi \mathrm{d} s\right| \leq\|B[u]\|_{L^{\infty}(Q)^{n}}\left(\frac{M}{2}\|\rho\|_{L^{2}(\Omega)}^{2}+\frac{1}{2 M}\|\nabla \varphi\|_{L^{2}(\Omega)^{n}}^{2}\right) .
$$

Thus

$$
\begin{aligned}
a[u(t)](\varphi, \varphi) & =\nu \int_{\Omega}|\nabla \varphi|^{2} \mathrm{~d} x+\int_{\Omega} \varphi B[u(t)] \cdot \nabla \varphi \mathrm{d} x \\
& \geq \nu\|\varphi\|_{H^{1}(\Omega)}^{2}-\nu\|\varphi\|_{L^{2}(\Omega)}^{2}-\|B[u]\|_{L^{\infty}(Q)^{n}}\left(\frac{M}{2}\|\varphi\|_{L^{2}(\Omega)}^{2}+\frac{1}{2 M}\|\nabla \varphi\|_{L^{2}(\Omega)^{n}}^{2}\right) \\
& \geq \gamma\|\varphi\|_{H^{1}(\Omega)}^{2}-\lambda\|\varphi\|_{L^{2}(\Omega)}^{2},
\end{aligned}
$$

if $M$ is chosen such that $\gamma:=\nu-\frac{\|B[u]\|_{L^{\infty}(Q)^{n}}}{2 M}$ is positive, and we set $\lambda:=\nu+\frac{M}{2}\|B[u]\|_{L^{\infty}(Q)^{n}}$.

We conclude from (2.11) and (2.12) that the hypotheses of boundedness and coercivity of $a[u]$ in LionsMagenes ([32], Thm. 4.1 and Rem. 4.3, pp. 238-239) are satisfied and, therefore, their result can be applied.

Now we extend the existence result to $L^{2}$-controls. The proof via Gronwall's Lemma is inspired by that of ([13], Prop. 2.1).

Theorem 2.6. For all $u \in L^{2}\left(0, T ; \mathbb{R}^{n}\right), f \in L^{2}\left(0, T ; H^{1}(\Omega)^{*}\right)$, and $\rho_{0} \in L^{2}(\Omega)$, the state equation (2.1)-(2.3) has a unique weak solution $\rho \in W(0, T)$. It obeys the estimate

$$
\|\rho\|_{W(0, T)}^{2} \leq C_{0}\left(\left\|\rho_{0}\right\|_{L^{2}(\Omega)}^{2}+\|f\|_{L^{2}\left(0, T ; H^{1}(\Omega)^{*}\right)}^{2}\right),
$$

where the constant $C_{0}$ depends continuously on $\|u\|_{2}$ but is independent of $f$ and $\rho_{0}$.

Proof. (i) First, we derive an a priori estimate for a solution $\rho$ of (2.1)-(2.3). To this aim, we introduce a new function $\eta$ by $\rho(t)=e^{\nu t} \eta(t)$ and transform the Fokker-Planck equation (2.1) into the equivalent equation

$$
\partial_{t} \eta-\nu \Delta \eta+\nu \eta-\operatorname{div}(\eta B[u])=e^{-\nu t} f
$$


Next, we test the weak formulation by $\varphi=\eta$. Select an arbitrary $t \in(0, T)$, and integrate over $(0, t)$ to get

$$
\int_{0}^{t} \frac{1}{2} \frac{\mathrm{d}}{\mathrm{d} s}\|\eta(s)\|_{L^{2}(\Omega)}^{2} \mathrm{~d} s+\nu \int_{0}^{t}\|\eta(s)\|_{H^{1}(\Omega)}^{2} \mathrm{~d} s+\int_{0}^{t} \int_{\Omega} \eta(s) B[u(s)] \cdot \nabla \eta(s) \mathrm{d} x \mathrm{~d} s=\int_{0}^{t} e^{-\nu s}\langle f(s), \eta(s)\rangle \mathrm{d} s .
$$

Notice that we have $|B[u](x, t)|=|u(t) \otimes b(x)+c(x)| \leq d_{1}(|u(t)|+1)$ a.e. in $Q$, since $b$ and $c$ are bounded. Applying Young's inequality in a standard way to the third term on the l.h.s. and to the term in the r.h.s. of latter display, and compensating the $H^{1}$-terms on $\eta$ on both sides of the inequality, we arrive at

$$
\begin{aligned}
\frac{1}{2}\|\eta(t)\|_{L^{2}(\Omega)}^{2}+\frac{\nu}{2} \int_{0}^{t}\|\eta(s)\|_{H^{1}(\Omega)}^{2} \mathrm{~d} s & \\
& \leq \frac{1}{\nu}\|f\|_{L^{2}\left(0, T ; H^{1}(\Omega)^{*}\right)}^{2}+\frac{1}{2}\left\|\rho_{0}\right\|_{L^{2}(\Omega)}^{2}+\int_{0}^{t}\|\eta(s)\|_{L^{2}(\Omega)}^{2} \frac{d_{1}^{2}}{\nu}(|u(s)|+1)^{2} \mathrm{~d} s .
\end{aligned}
$$

By Gronwall's inequality for $z(t):=\|\eta(t)\|_{L^{2}(\Omega)}^{2}$ and by the estimate $(|u|+1)^{2} \leq 2\left(|u|^{2}+1\right)$, the inequality

$$
\|\eta(t)\|_{L^{2}(\Omega)}^{2} \leq\left(\frac{2}{\nu}\|f\|_{L^{2}\left(0, T ; H^{1}(\Omega)^{*}\right)}^{2}+\left\|\rho_{0}\right\|_{L^{2}(\Omega)}^{2}\right) \exp \left(\int_{0}^{t} \frac{4 d_{1}^{2}}{\nu}\left(|u(s)|^{2}+1\right) \mathrm{d} s\right)
$$

is deduced. Therefore, since $t \in(0, T)$ was taken arbitrarily,

$$
\|\eta\|_{L^{\infty}\left(0, T ; L^{2}(\Omega)\right)}^{2} \leq d_{2}\left(\|f\|_{L^{2}\left(0, T ; H^{1}(\Omega)^{*}\right)}^{2}+\left\|\rho_{0}\right\|_{L^{2}(\Omega)}^{2}\right)
$$

follows in turn, where $d_{2}$ depends continuously on $\|u\|_{2}$. Inserting this estimate in (2.15), an analogous inequality for $\|\eta\|_{L^{2}\left(0, T ; H^{1}(\Omega)\right)}$ is derived that implies an estimate for $\left\|\partial_{t} \eta\right\|_{L^{2}\left(0, T ; H^{1}(\Omega)^{*}\right)}$ in a standard way. Altogether, this finally permits to deduce that

$$
\|\eta\|_{W(0, T)} \leq c_{3}\left(\|f\|_{L^{2}\left(0, T ; H^{1}(\Omega)^{*}\right)}^{2}+\left\|\rho_{0}\right\|_{L^{2}(\Omega)}^{2}\right),
$$

where $c_{3}$ depends continuously on $\|u\|_{2}$. Transforming back by $\rho=e^{\nu t} \eta$ finally yields (2.13) as a priori estimate.

(ii) Now we fix an arbitrary $u \in L^{2}\left(0, T ; \mathbb{R}^{n}\right)$ and show that $(2.1)-(2.3)$ has a solution. To this end, we select a sequence $\left\{u_{k}\right\} \subset L^{\infty}\left(0, T ; \mathbb{R}^{n}\right)$ such that $\left\|u_{k}-u\right\|_{2} \rightarrow 0$ for $k \rightarrow \infty$. We can assume that $\left\|u_{k}\right\|_{2} \leq 2\|u\|_{2}$, for all $k \in \mathbb{N}$.

Thanks to Theorem 2.5, to each $u_{k}$, a unique solution $\rho_{k} \in W(0, T)$ of $(2.1)-(2.3)$ exists. By the a priori estimate (2.16) obtained in (i) above, the sequence $\left\{\rho_{k}\right\}$ is bounded in $W(0, T)$ and we can select a subsequence that converges weakly to some $\rho \in W(0, T)$. Possibly after renumbering, we can assume that $\rho_{k} \rightarrow \rho$.

Passing to the limit in the weak formulation (2.10), it is easy to confirm that $\rho$ solves (2.1)-(2.3). Here, the term $\rho_{k} u_{k} \otimes b \cdot \nabla \varphi$ needs some special care. Let us confirm that $\rho_{k} u_{k} \otimes b$ converges weakly to $\rho u \otimes b$ in $L^{2}\left(Q ; \mathbb{R}^{n}\right)$. Indeed, for all $v \in L^{2}\left(Q ; \mathbb{R}^{n}\right)$,

$$
\iint_{Q} \rho_{k}\left(u_{k} \otimes b\right) \cdot v \mathrm{~d} x \mathrm{~d} t=\int_{0}^{T} u_{k} \cdot\left(\int_{\Omega} \rho_{k} b \otimes v \mathrm{~d} x\right) \mathrm{d} t \rightarrow \int_{0}^{T} u \cdot\left(\int_{\Omega} \rho b \otimes v \mathrm{~d} x\right) \mathrm{d} t, \quad k \rightarrow \infty,
$$

since $\rho_{k} \rightarrow \rho$ in $L^{\infty}\left(0, T ; L^{2}(\Omega)\right)$ follows from the weak convergence of $\left\{\rho_{k}\right\}$ to $\rho$ in $W(0, T)$, from the continuous embedding of this space in $C\left([0, T], L^{2}(\Omega)\right)$, and from the fact that the mapping $\rho \mapsto \int_{\Omega} \rho b \otimes v \mathrm{~d} x$ is linear and continuous from $L^{\infty}\left(0, T ; L^{2}(\Omega)\right)$ to $L^{2}\left(0, T ; \mathbb{R}^{n}\right)$. The uniqueness follows in a standard way by the a priori estimates. 
Remark 2.7 (On the mass conservation and nonnegativity of the solutions). Note that, by choosing $\varphi \equiv 1$ in (2.6), it follows that

$$
\int_{\Omega} \rho(x, t) \mathrm{d} x=\int_{\Omega} \rho_{0}(x) \mathrm{d} s \quad \text { for a.a. } t \in(0, T),
$$

where $\rho_{0}$ is the initial condition and $\rho$ the corresponding weak solution of (2.1)-(2.3). Moreover, from Chipot ([19], Thm. 11.9, p. 202) a Weak Maximum Principle for the weak solutions of (2.1)-(2.3) follows, from which we can deduce, in particular, that weak solutions are nonnegative whenever the initial condition is nonnegative.

\section{The optimal CONTROL PROBLEM}

In our optimal control problem, we minimize the cost functional

$$
J(\rho, u):=\frac{\alpha_{Q}}{2} \int_{0}^{T}\left\|\rho(t)-\rho_{Q}(t)\right\|_{L^{2}(\Omega)}^{2} \mathrm{~d} t+\frac{\alpha_{\Omega}}{2}\left\|\rho(T)-\rho_{\Omega}\right\|_{L^{2}(\Omega)}^{2}+\sum_{i=1}^{n}\left(\frac{\gamma_{i}}{2}\left\|u_{i}\right\|_{2}^{2}+\beta_{i} \int_{0}^{T} u_{i}(t) \mathrm{d} t\right),
$$

with $\rho_{Q} \in L^{2}(Q), \rho_{\Omega} \in L^{2}(\Omega)$, and $\beta_{i}, \gamma_{i} \geq 0$ for $i=1, \ldots, n$, subject to the control constraints

$$
u^{\min }(t) \leq u(t) \leq u^{\max }(t) \quad \text { for a.e. } t \in[0, T]
$$

where the inequalities are defined componentwise, and the bounds $u^{\min }, u^{\max }$ belong to $L^{\infty}\left(0, T ; \mathbb{R}^{n}\right)$. The set of admissible controls is

$$
\mathcal{U}_{\mathrm{ad}}:=\left\{u \in L^{\infty}\left(0, T ; \mathbb{R}^{n}\right):(3.2) \text { holds }\right\}
$$

The parameters $\beta_{i}, \gamma_{i}, i=1, \ldots, n$, are allowed to vanish simultaneously unless second order sufficient optimality conditions are investigated. Then, all $\gamma_{i}$ have to be positive.

Definition 3.1. Let us define the control-to-state mapping

$$
G: L^{2}\left(0, T ; \mathbb{R}^{n}\right) \rightarrow W(0, T),
$$

that associates to each $u \in L^{2}\left(0, T ; \mathbb{R}^{n}\right)$ the unique weak solution $\rho \in W(0, T)$ of (2.1)-(2.3). When necessary, we may write $G(u)$ to denote the state $\rho$ corresponding to $u$.

Our optimal control problem can be rewritten as

$$
\min _{u \in \mathcal{U}_{\mathrm{ad}}} J(G(u), u)
$$

We study two types of solutions, that we define next. We say that $\bar{u} \in \mathcal{U}_{\text {ad }}$ is an $L^{\infty}$-local solution (resp., $L^{2}$-local solution) of $(\mathrm{P})$ if there exists $\varepsilon>0$ such that $J(G(\bar{u}), \bar{u}) \leq J(G(u), u)$ holds for every $u \in \mathcal{U}_{\text {ad }} \cap B_{\varepsilon}^{\infty}(\bar{u})$ (resp., $u \in \mathcal{U}_{\text {ad }} \cap B_{\varepsilon}^{2}(\bar{u})$ ), where $B_{\varepsilon}^{\infty}(\bar{u})$ denotes the open ball in $L^{\infty}\left(0, T ; \mathbb{R}^{n}\right)$ (resp., in $L^{2}\left(0, T ; \mathbb{R}^{n}\right)$ ) of radius $\varepsilon$ centered at $\bar{u}$.

\subsection{Existence of optimal controls}

Theorem 3.2 (Existence of an optimal control). There exists (at least) one optimal control for (P).

Proof. In view of the control constraints (3.2), the set of admissible controls $\mathcal{U}_{\text {ad }}$ is bounded in $L^{\infty}\left(0, T ; \mathbb{R}^{n}\right)$. Hence, thanks to the estimate (2.13), the set of states associated to admissible controls is bounded in $W(0, T)$. 
Therefore, the cost functional $J$ is bounded from below on the set of admissible state-control pairs. Thus, there exists a minimizing sequence $\left\{\left(\rho_{k}, u_{k}\right)\right\} \subset W(0, T) \times \mathcal{U}_{\text {ad }}$, where $\rho_{k}:=G\left(u_{k}\right)$, such that

$$
J\left(\rho_{k}, u_{k}\right) \longrightarrow \inf _{u \in \mathcal{U}_{\mathrm{ad}}} J(G(u), u) .
$$

Since $\left\{u_{k}\right\}$ is bounded in $L^{\infty}\left(0, T ; \mathbb{R}^{n}\right)$, it contains a weakly* converging subsequence, thus, keeping the same index, we have

$$
u_{k} \stackrel{*}{\rightarrow} \bar{u} \quad \text { in } L^{\infty}\left(0, T ; \mathbb{R}^{n}\right)
$$

for some $\bar{u} \in L^{\infty}\left(0, T ; \mathbb{R}^{n}\right)$. The corresponding sequence of states $\left\{\rho_{k}\right\}$ forms a bounded sequence in $W(0, T)$. Thus, there exists $\bar{\rho} \in W(0, T)$ such that (extracting if necessary a subsequence)

$$
\rho_{k} \rightarrow \bar{\rho} \quad \text { in } W(0, T)
$$

The objective functional $J$ is weakly lower semicontinuous, so we obtain that

$$
J(\bar{\rho}, \bar{u}) \leq \liminf _{k \rightarrow \infty} J\left(\bar{\rho}_{k}, \bar{u}_{k}\right)
$$

and hence, $\bar{u}$ is optimal provided that $\bar{\rho}$ is the associated state.

The main work of the proof is to show that $\bar{\rho}$ is the state associated to $\bar{u}$, and then $J(\bar{\rho}, \bar{u})=$ $\inf _{u \in \mathcal{U}_{\text {ad }}} J(G(u), u)$. For this, we prove that we can pass to the limit in (2.10).

Using Aubin-Lions' Lemma [11], we can deduce from (3.4) that $\left\{\rho_{k}\right\}$ has a subsequence converging to $\bar{\rho}$ strongly in $L^{2}(Q)$. This is, keeping the same index for the subsequence,

$$
\rho_{k} \longrightarrow \bar{\rho} \quad \text { (strongly) in } L^{2}(Q) \text {. }
$$

Putting all together, we have that $\rho_{k} \rightarrow \bar{\rho}$ weakly in $L^{2}\left(0, T ; H^{1}(\Omega)\right), \partial_{t} \rho_{k} \rightarrow \partial_{t} \bar{\rho}$ weakly in $L^{2}\left(0, T ; H^{1}(\Omega)^{*}\right)$, and $\rho_{k} \rightarrow \bar{\rho}$ strongly in $L^{2}(Q)$.

We next show that $\bar{\rho}=G(\bar{u})$.

Given $\varphi \in W_{2}^{1,1}(Q)$, with $\varphi(\cdot, T)=0$, we have

$$
\int_{0}^{T}\left(\left\langle\partial_{t} \rho_{k}(t), \varphi(t)\right\rangle+\int_{\Omega} \nu \nabla \rho_{k}(t) \cdot \nabla \varphi(t) \mathrm{d} x\right) \mathrm{d} t \rightarrow \int_{0}^{T}\left(\left\langle\partial_{t} \bar{\rho}(t), \varphi(t)\right\rangle+\int_{\Omega} \nu \nabla \bar{\rho}(t) \cdot \nabla \varphi(t) \mathrm{d} x\right) \mathrm{d} t .
$$

In the weak formulation of the state equation, it only remains to check the convergence of the part containing $B\left[u_{k}\right]$. To this aim, for $i=1, \ldots, n$, we consider the terms

$$
\rho_{k} B_{i}\left[u_{k}\right] \frac{\partial \varphi}{\partial x_{i}}=\rho_{k}\left(c_{i}+b_{i} u_{k, i}\right) \frac{\partial \varphi}{\partial x_{i}} .
$$

It is easy to see that $\rho_{k} c_{i} \frac{\partial \varphi}{\partial x_{i}} \rightarrow \bar{\rho} c_{i} \frac{\partial \varphi}{\partial x_{i}}$ and $\rho_{k} b_{i} \frac{\partial \varphi}{\partial x_{i}} \rightarrow \bar{\rho} b_{i} \frac{\partial \varphi}{\partial x_{i}}$ strongly in $L^{1}(Q)$, since $\rho_{k} \rightarrow \bar{\rho}$ strongly in $L^{2}(Q), c_{i}, b_{i} \in L^{\infty}(\Omega)$ and $\frac{\partial \varphi}{\partial x_{i}} \in L^{2}(Q)$. Therefore, in view of the weak* convergence of $\left\{u_{k}\right\}$ to $\bar{u}$, we get that

$$
\iint_{Q} u_{k} \rho_{k} b_{i} \frac{\partial \varphi}{\partial x_{i}} \mathrm{~d} x \mathrm{~d} t \rightarrow \iint_{Q} \bar{u} \bar{\rho} b_{i} \frac{\partial \varphi}{\partial x_{i}} \mathrm{~d} x \mathrm{~d} t, \quad \text { for } k \rightarrow \infty .
$$

Thus, $\bar{\rho}$ is the state associated to $\bar{u}$. This concludes the proof. 
We are not able to prove uniqueness of optimal controls, since the control-to-state mapping is nonlinear. Therefore, the reduced objective functional is nonconvex in general.

\section{Properties of the CONTROL-TO-STAte MAPPing}

In this section, we prove Fréchet differentiability of the control-to-state mapping $G$ and derive the first order necessary optimality condition of Proposition 4.5 as a corollary.

\subsection{Fréchet differentiability of the control-to-state mapping}

We start by proving the differentiability of $G$ by the Implicit Function Theorem. To this aim, we define the mapping

$$
(\rho, u) \longmapsto \mathcal{G}(\rho, u):=\left(\partial_{t} \rho+a[u](\rho, \cdot), \rho(0)-\rho_{0}\right)
$$

from $W(0, T) \times L^{2}\left(0, T ; \mathbb{R}^{n}\right)$ to $L^{2}\left(0, T ; H^{1}(\Omega)^{*}\right) \times L^{2}(\Omega)$. The state equation can be viewed as the equation

$$
\mathcal{G}(\rho, u)=0 .
$$

Proposition 4.1. The mapping $\mathcal{G}$ is of class $C^{\infty}$.

Proof. The first component $\mathcal{G}_{1}$ of $\mathcal{G}$ is defined by

$$
\mathcal{G}_{1}(\rho, u)(\varphi):=\int_{0}^{T}\left\langle\partial_{t} \rho(t), \varphi(t)\right\rangle \mathrm{d} t+\iint_{Q} \nabla \rho \cdot \nabla \varphi \mathrm{d} x \mathrm{~d} t+\iint_{Q} \rho B[u] \cdot \nabla \varphi \mathrm{d} x \mathrm{~d} t .
$$

Its first two summands clearly define linear and continuous mappings from $W(0, T)$ to $L^{2}\left(0, T ; H^{1}(\Omega)^{*}\right)$, hence they are of class $C^{\infty}$. Therefore, it suffices to confirm that the operator

$$
(\rho, u) \mapsto \rho B[u]
$$

is of class $C^{\infty}$ from $W(0, T) \times L^{2}\left(0, T ; \mathbb{R}^{n}\right)$ to $L^{2}\left(Q ; \mathbb{R}^{n}\right)$. This, however, follows easily from the quadratic nature of $\rho B[u]$. For increments $\sigma \in W(0, T)$ and $v \in L^{2}\left(0, T ; \mathbb{R}^{n}\right)$, we have

$$
(\rho+\sigma) B[u+v]=(\rho+\sigma)(c+b \otimes(u+v))=\rho B[u]+\rho b \otimes v+\sigma(c+b \otimes u)+\sigma b \otimes v .
$$

This is a second order Taylor expansion of the mapping $(\rho, u) \mapsto \rho B[u]$ with continuous linear and quadratic parts, and vanishing remainder term. Let us exemplarily confirm the continuity of the quadratic term. We have

$$
\|\sigma b \otimes v\|_{L^{2}(Q)^{n}} \leq\|b\|_{L^{\infty}(\Omega)^{n}}\|\sigma\|_{C\left([0, T] ; L^{2}(\Omega)\right)}\|v\|_{2} \leq c\|\sigma\|_{W(0, T)}\|v\|_{2},
$$

hence the continuity of the quadratic form $\sigma b \otimes v$. Therefore $\mathcal{G}_{1}$ is of class $C^{\infty}$. The second component of $\mathcal{G}$ is obviously of class $C^{\infty}$.

We thank the anonymous referee, who pointed out that our first proof in [8] already covered the differentiability of $\mathcal{G}$ with respect to $u$ in $L^{2}\left(0, T ; \mathbb{R}^{n}\right)$. This paved the way for proving the differentiability of $G$ in $L^{2}\left(0, T ; \mathbb{R}^{n}\right)$ rather than in $L^{\infty}\left(0, T ; \mathbb{R}^{n}\right)$ as in $[8]$.

Corollary 4.2. The control-to-state mapping $G: L^{2}\left(0, T ; \mathbb{R}^{n}\right) \rightarrow W(0, T)$ is of class $C^{\infty}$.

Proof. In view of Proposition 4.1, we have that $\mathcal{G}$ is of class $C^{\infty}$ and that

$$
\partial_{\rho} \mathcal{G}(\rho, u) z=\left(\partial_{t} z+a[u](z, \cdot), z(0)\right) .
$$


Given $u \in L^{2}\left(0, T ; \mathbb{R}^{n}\right), f \in L^{2}\left(0, T ; H^{1}(\Omega)^{*}\right)$ and $z_{0} \in L^{2}(\Omega)$, the system

$$
\begin{aligned}
\partial_{t} z+a[u](z, \cdot) & =f, \\
z(0) & =z_{0},
\end{aligned}
$$

is again a Fokker-Planck equation as the state equation and, therefore, it has a unique (weak) solution $z\left[z_{0}, f\right]$ that belongs to $W(0, T)$, and depends continuously on $z_{0} \in L^{2}(\Omega)$ and on $f \in L^{2}\left(0, T ; H^{1}(\Omega)^{*}\right)$. Therefore, thanks to the existence and uniqueness Theorem 2.6, $\partial_{\rho} \mathcal{G}(\rho, u)$ is an isomorphism from $W(0, T)$ to $L^{2}\left(0, T ; H^{1}(\Omega)^{*}\right) \times L^{2}(\Omega)$. Thus, the hypotheses of the Implicit Function Theorem are satisfied, and then $\mathcal{G}(\rho, u)=0$ implicitly defines the control-to-state operator $G: u \mapsto \rho$ that is itself of class $C^{\infty}$.

In the next proposition and for other occasions throughout the remainder of the article, we will use the following type of functional: for given $w \in L^{2}\left(\Omega ; \mathbb{R}^{n}\right)$, we introduce $\mathrm{d}[w] \in H^{1}(\Omega)^{*}$ defined by

$$
\mathrm{d}[w](\varphi):=-\int_{\Omega} w \cdot \nabla \varphi \mathrm{d} x
$$

for all $\varphi \in H^{1}(\Omega)$.

Proposition 4.3. Let $u \in L^{2}\left(0, T ; \mathbb{R}^{n}\right)$ and $\rho:=G(u)$. For any $v \in L^{2}\left(0, T ; \mathbb{R}^{n}\right)$, we have that $G^{\prime}(u) v=z$, where $z$ belongs to $W(0, T)$ and is the weak solution of the linearized state equation at $(\rho, u)$ that is given by

$$
\begin{aligned}
\partial_{t} z+a[u](z, \cdot) & =\mathrm{d}[\rho b \otimes v], \\
z(0) & =0 .
\end{aligned}
$$

Moreover, the following estimate holds,

$$
\|z\|_{C\left([0, T] ; L^{2}(\Omega)\right)}+\|z\|_{W(0, T)} \leq K\|b\|_{L^{\infty}(\Omega)^{n}}\|\rho\|_{C\left([0, T] ; L^{2}(\Omega)\right)}\|v\|_{2},
$$

where $K$ depends continuously on $\|u\|_{2}$ but does not depend on $v$.

Proof. The fact that (4.4) possesses a unique weak solution $z$ in $W(0, T)$ follows from Theorem 2.6 as already observed in the proof of Corollary 4.2 above. The representation $G^{\prime}(u) v=z$ follows from a direct application of the Implicit Function Theorem by differentiating the state equation (2.1)-(2.3) with respect to the control.

It remains to estimate the $L^{2}\left(0, T ; H^{1}(\Omega)^{*}\right)$-norm of the r.h.s. function $\mathrm{d}[\rho b \otimes v]$, in order to apply the estimates of Theorem 2.6. Take any $\varphi \in H^{1}(\Omega)$; then for a.a. $t \in[0, T]$ we have

$$
|\mathrm{d}[\rho(t) b \otimes v(t)](\varphi)|=\left|\int_{\Omega} \rho(t)(b \otimes v(t)) \cdot \nabla \varphi \mathrm{d} x\right| \leq \sum_{i=1}^{n}\left|v_{i}(t)\right|\left\|b_{i}\right\|_{L^{\infty}(\Omega)}\|\rho(t)\|_{L^{2}(\Omega)}\|\varphi\|_{H^{1}(\Omega)} .
$$

Thus, for a.e. $t \in[0, T]$,

$$
\|\mathrm{d}[\rho(t) b \otimes v(t)]\|_{H^{1}(\Omega)^{*}} \leq\|b\|_{L^{\infty}(\Omega)^{n}}\|\rho(t)\|_{L^{2}(\Omega)} \sum_{i=1}^{n}\left|v_{i}(t)\right|
$$

and hence

$$
\|\mathrm{d}[\rho b \otimes v]\|_{L^{2}\left(0, T ; H^{1}(\Omega)^{*}\right)} \leq M\|b\|_{L^{\infty}(\Omega)^{n}}\|\rho\|_{L^{2}\left(0, T ; L^{2}(\Omega)\right)}\|v\|_{2},
$$


for some constant $M$ depending on the control dimension $n$. The estimate (4.5) and the continuous dependence of $K$ on $\|u\|_{2}$ follow from Theorem 2.6 with $f:=\mathrm{d}[\rho b \otimes v]$ and $\rho_{0}=0$. This concludes the proof.

The linearized state equation $(4.4)$ at $(\rho, u)$ in the direction of $v$ is to be understood as

$$
\begin{aligned}
\int_{\Omega}\left(\partial_{t} z \varphi+(\nu \nabla z+z B[u]) \cdot \nabla \varphi\right) \mathrm{d} x & =-\int_{\Omega} \rho(b \otimes v) \cdot \nabla \varphi \mathrm{d} x \text { for all } \varphi \in H^{1}(\Omega), \\
z(0) & =0,
\end{aligned}
$$

or, in the strong form:

$$
\begin{array}{rlrl}
\partial_{t} z-\nu \Delta z-\operatorname{div}(z B[u]) & =\operatorname{div}(\rho(b \otimes v)) & \text { in } Q, \\
(\nu \nabla z+z B[u]) \cdot n & =-(\rho b \otimes v) \cdot n \quad \text { in } \Sigma, \\
z(0) & =0 \quad \text { in } \Omega . &
\end{array}
$$

Remark 4.4. Following Ladyzhenskaya et al. [30], an equivalent variational formulation of the linearized state equation (4.6) is given as follows: $z$ is a function in $W_{2}^{1,0}(Q)$ such that

$$
\iint_{Q}\left(-z \partial_{t} \varphi+(\nu \nabla z+z B[u]) \cdot \nabla \varphi\right) \mathrm{d} x \mathrm{~d} t=-\iint_{Q} \rho(b \otimes v) \cdot \nabla \varphi \mathrm{d} x \mathrm{~d} t \quad \text { for all } \varphi \in W_{2}^{1,1}(Q) \text { with } \varphi(\cdot, T)=0
$$

The additional property that $z \in W(0, T)$ is a standard consequence of (4.8).

Let us define the reduced cost functional as

$$
F(u):=J(G(u), u)
$$

By the chain rule, the reduced cost functional $F$ is continuously Fréchet differentiable, since $J$ and $G$ have this property.

Proposition 4.5 (First order necessary condition). If $\bar{u}$ is an $L^{\infty}$-local minimum for $(\mathrm{P})$, then

$$
F^{\prime}(\bar{u})(u-\bar{u}) \geq 0 \quad \text { for every } u \in \mathcal{U}_{\mathrm{ad}}
$$

Proof. The proof is standard and follows straightforwardly by writing the Newton quotient of $F$.

The variational inequality (4.9) also holds for $L^{2}$-local minima, because any $L^{2}$-local minimum is also an $L^{\infty}$-local one.

\subsection{Lipschitz continuity of the control-to-state mapping}

We conclude this section by proving the local Lipschitz continuity of $G$ that mainly follows from its differentiability. However, we are particularly interested in the continuous dependence of the Lipschitz constant on the control.

Proposition 4.6. The control-to-state mapping $G$ is locally Lipschitz, i.e. for any pair $u_{1}, u_{2} \in L^{2}\left(0, T ; \mathbb{R}^{n}\right)$ with associated states $\rho_{1}:=G\left(u_{1}\right), \rho_{2}:=G\left(u_{2}\right)$, one has

$$
\left\|\rho_{2}-\rho_{1}\right\|_{W(0, T)}^{2} \leq C\left\|\rho_{1}\right\|_{C\left([0, T] ; L^{2}(\Omega)\right)}^{2}\left\|u_{2}-u_{1}\right\|_{2}^{2},
$$

where $C$ depends continuously on $\left\|u_{2}\right\|_{2}$. 
Proof. Let us consider two weak solutions $\rho_{1}, \rho_{2}$ of (2.1)-(2.3), associated to $u_{1}, u_{2}$, respectively. Setting $\delta \rho:=$ $\rho_{2}-\rho_{1}$, we have, for any $\varphi \in H^{1}(\Omega)$,

$$
\frac{\mathrm{d}}{\mathrm{d} t} \int_{\Omega}(\delta \rho) \varphi \mathrm{d} x+\int_{\Omega}\left(\nu \nabla(\delta \rho)+(\delta \rho) B\left[u_{2}\right]\right) \cdot \nabla \varphi \mathrm{d} x=-\int_{\Omega} \rho_{1} b \otimes\left(u_{2}-u_{1}\right) \cdot \nabla \varphi \mathrm{d} x .
$$

The latter is equivalent to the linearized equation (4.6) for $z=\delta \rho$ and $v=u_{2}-u_{1}$. Applying the estimate (4.5) yields the desired result.

Notice that (4.10) implies an analogous inequality for $\left\|\rho_{2}-\rho_{1}\right\|_{C\left([0, T] ; L^{2}(\Omega)\right)}$ since $W(0, T)$ is continuously embedded in $C\left([0, T] ; L^{2}(\Omega)\right)$. The constant neither depends on $\left\|u_{2}\right\|_{\infty}$ as well, since the embedding constant does not depend on the control.

\section{THE ADJOINT EQUATION}

\subsection{Definition of the adjoint equation}

By an adjoint state, the variational inequality (4.9) can be transformed to a more convenient form. To this aim, we introduce the following adjoint equation for the adjoint state $p$ associated with $(\rho, u)$ :

$$
\begin{aligned}
-\partial_{t} p-\nu \Delta p+B[u] \cdot \nabla p & =\alpha_{Q}\left(\rho-\rho_{Q}\right) \quad \text { in } Q, \\
p(T) & =\alpha_{\Omega}\left(\rho(T)-\rho_{\Omega}\right) \quad \text { in } \Omega, \\
\partial_{n} p & =0 \quad \text { on } \Sigma .
\end{aligned}
$$

The form of the adjoint equation can be found e.g. by application of a formal Lagrangian technique, cf. [38], chapter 2.6. In weak formulation, the adjoint equation at $(\rho, u)$ is defined by

$$
\begin{aligned}
\int_{\Omega}\left(-\varphi \partial_{t} p+\nu \nabla p \cdot \nabla \varphi+\varphi B[u] \cdot \nabla p\right) \mathrm{d} x & =\alpha_{Q} \int_{\Omega}\left(\rho-\rho_{Q}\right) \varphi \mathrm{d} x \quad \text { on } \mathcal{D}^{\prime}(0, T) \text { for all } \varphi \in H^{1}(\Omega), \\
p(T) & =\alpha_{\Omega}\left(\rho(T)-\rho_{\Omega}\right) .
\end{aligned}
$$

The unique (weak) solution $p$ of (5.1)-(5.3) is called the adjoint state associated with $(\rho, u)$. Note that, with $f:=\alpha_{Q}\left(\rho-\rho_{Q}\right),(5.4)$ can be rewritten as

$$
\begin{aligned}
-\partial_{t} p+a[u(\cdot)](\cdot, p) & =f \quad \text { in } L^{2}\left(0, T ; H^{1}(\Omega)^{*}\right), \\
p(T) & =\alpha_{\Omega}\left(\rho(T)-\rho_{\Omega}\right),
\end{aligned}
$$

provided that $p$ enjoys the higher regularity $p \in W(0, T)$.

For the weak formulation we will also use the definition of Ladyzhenskaya et al. [30], because for given $u \in L^{2}\left(0, T ; \mathbb{R}^{n}\right)$ we were only able to show the regularity $p \in W_{2}^{1,0}(Q)$, namely

$$
\begin{gathered}
\iint_{Q}\left(p \partial_{t} \varphi+\nu \nabla p \cdot \nabla \varphi+\varphi B[u] \cdot \nabla p\right) \mathrm{d} x \mathrm{~d} t=-\int_{\Omega} \alpha_{\Omega}\left(\rho(T)-\rho_{\Omega}\right) \varphi(T) \mathrm{d} x+\alpha_{Q} \iint_{Q}\left(\rho-\rho_{Q}\right) \varphi \mathrm{d} x \mathrm{~d} t \\
\text { for all } \varphi \in W_{2}^{1,1}(Q) \text { with } \varphi(\cdot, 0)=0 .
\end{gathered}
$$

Proposition 5.1. Given $u \in L^{\infty}\left(0, T ; \mathbb{R}^{n}\right)$ and $f \in L^{2}\left(0, T ; H^{1}(\Omega)^{*}\right)$, equation (5.1)-(5.3), with right-hand side $\alpha_{Q}\left(\rho-\rho_{Q}\right)$ of (5.1) replaced by the general function $f$, has a unique weak solution $p \in W(0, T)$ and the 
following estimate holds,

$$
\|p\|_{W(0, T)} \leq C\left(\left\|\alpha_{\Omega}\left(\rho(T)-\rho_{\Omega}\right)\right\|_{L^{2}(\Omega)}+\|f\|_{L^{2}\left(0, T ; H^{1}(\Omega)^{*}\right)}\right),
$$

where $\rho:=G(u)$ and $C$ depends continuously on $\|u\|_{2}$. In particular, when $f=\alpha_{Q}\left(\rho-\rho_{Q}\right)$, one has

$$
\|p\|_{W(0, T)} \leq C\left(\left\|\alpha_{\Omega}\left(\rho(T)-\rho_{\Omega}\right)\right\|_{L^{2}(\Omega)}+\left\|\alpha_{Q}\left(\rho-\rho_{Q}\right)\right\|_{L^{2}(Q)}\right) .
$$

Proof. We first apply the transformation of time $\tau=T-t$ and $\tilde{p}(\tau)=p(T-\tau)$. Then the equation is transformed to a forward one. In particular, the terminal condition for $p$ becomes an initial condition for $\tilde{p}$. Then we proceed as in the proof of Theorem 2.5. It is easy to confirm that the estimates (2.11) and (2.12) remain true for the choice $\rho:=\varphi$ and $\varphi:=p$, where $\varphi \in L^{2}\left(0, T, H^{1}(\Omega)\right)$ is the test function and $p \in L^{2}\left(0, T, H^{1}(\Omega)\right)$ is the desired solution. Notice that $p$ appears through a gradient. Now the existence of $p \in W(0, T)$ follows again from the result by Lions and Magenes [32].

Remark 5.2. For $u \in L^{2}\left(0, T ; \mathbb{R}^{n}\right)$, we obtain the existence of an adjoint state $p \in W_{2}^{1,0}(Q)$ that satisfies the weak formulation (5.6). This complies with ([30], Thm.4.1, p.153) that ensures a solution $p \in W_{2}^{1,0}(Q) \cap$ $L^{\infty}\left(0, T ; L^{2}(\Omega)\right)$. To confirm the existence of $p$ in $W_{2}^{1,0}(Q)$, we proceed as in the proof of Theorem 2.6 and approximate $u$ by a sequence of controls $\left\{u_{k}\right\} \subset L^{\infty}\left(0, T ; \mathbb{R}^{n}\right)$. An associated subsequence of adjoint states $p_{k}$ converges weakly in $W_{2}^{1,0}(Q) \cong L^{2}\left(0, T ; H^{1}(\Omega)\right)$ to some $p \in W_{2}^{1,0}(Q)$, hence $\nabla p_{k}$ converges weakly in $L^{2}\left(0, T ; L^{2}(\Omega)^{n}\right)$ to $\nabla p$. Again, the bilinear term is the delicate point: as in (5.6), we are allowed to use more regular test functions $\varphi \in W_{2}^{1,1}(Q) \subset C\left([0, T] ; L^{2}(\Omega)\right)$. We deduce

$$
\iint_{Q} \varphi u_{k} \otimes b \cdot \nabla p_{k} \mathrm{~d} x \mathrm{~d} t \rightarrow \iint_{Q} \varphi u \otimes b \cdot \nabla p \mathrm{~d} x \mathrm{~d} t, \quad k \rightarrow \infty .
$$

Here, we take advantage of the weak convergence $u_{k} \otimes b \cdot \nabla p_{k} \rightarrow u \otimes b \cdot \nabla p$ in $L^{1}\left(0, T ; L^{2}(\Omega)\right)$ that fits to the regularity $\varphi \in C\left([0, T] ; L^{2}(\Omega)\right)$.

We were not able to prove that $p$ belongs to $W(0, T)$. The reason is that, in contrast to what occurs for the state equation, here the mapping

$$
\varphi \mapsto \iint_{Q} \varphi u \otimes b \cdot \nabla p \mathrm{~d} x \mathrm{~d} t
$$

is not continuous on $L^{2}\left(0, T ; H^{1}(\Omega)\right)$ for $u \in L^{2}\left(0, T ; \mathbb{R}^{n}\right)$; notice that we only know $\nabla p \in L^{2}\left(Q ; \mathbb{R}^{n}\right)$. Therefore, this linear functional does not belong to $L^{2}\left(0, T ; H^{1}(\Omega)^{*}\right)$.

We should mention that, even for $p \in W(0, T)$, the integral (5.10) is not well defined with test functions $\varphi \in L^{2}\left(0, T ; H^{1}(\Omega)\right)$, since we only know that $\nabla p \in L^{2}\left(0, T ; L^{2}(\Omega)^{n}\right)$. We would need the additional regularity $\nabla p \in L^{\infty}\left(0, T ; L^{2}(\Omega)^{n}\right)$, if $u \in L^{2}\left(0, T ; \mathbb{R}^{n}\right)$. We did not try to prove this, since our controls are essentially bounded. Therefore, in what follows we concentrate on the case of bounded controls. The main result of our paper, the second-order sufficient optimality conditions, is not influenced by this restriction. For a similar setting but for infinite horizon control, in ([14], Prop. 4.8) the authors were able to show that $p$ is in $W(0, T)$.

\subsection{First order necessary optimality conditions in terms of the adjoint state}

In this subsection we rewrite the first order condition of Proposition 4.5 in terms of the adjoint state. For this, we show the following technical result.

Lemma 5.3. Assume that $u \in L^{\infty}\left(0, T ; \mathbb{R}^{n}\right)$ is given and let $\rho=G(u)$ be its associated state. Let $z$ be the weak solution of the linearized state equation (4.4) corresponding to $v \in L^{2}\left(0, T ; \mathbb{R}^{n}\right)$, and let $p \in W(0, T)$ be the weak 
solution of the adjoint equation (5.1)-(5.3). Then

$$
\alpha_{Q} \int_{0}^{T} \int_{\Omega}\left(\rho-\rho_{Q}\right) z \mathrm{~d} x \mathrm{~d} t+\alpha_{\Omega} \int_{\Omega}\left(\rho(T)-\rho_{\Omega}\right) z(T) \mathrm{d} x=-\int_{0}^{T} \int_{\Omega} \rho(b \otimes v) \cdot \nabla p \mathrm{~d} x \mathrm{~d} t .
$$

Proof. It follows by testing the linearized equation (4.6) with $\varphi:=p$ and the adjoint equation (5.4) with $\varphi:=z$, and subsequent integration by parts (a detailed proof can be found in [8]).

Let us introduce the notation

$$
\Phi_{i}(t):=-\int_{\Omega} \rho(t) b_{i} \frac{\partial p(t)}{\partial x_{i}} \mathrm{~d} x+\gamma_{i} u_{i}(t)+\beta_{i} \quad \text { for } i=1, \ldots, n .
$$

Theorem 5.4. For any $u \in L^{\infty}\left(0, T ; \mathbb{R}^{n}\right)$ and $v \in L^{2}\left(0, T ; \mathbb{R}^{n}\right)$, one has

$$
F^{\prime}(u) v=\sum_{i=1}^{n} \int_{0}^{T} \Phi_{i}(t) v_{i}(t) \mathrm{d} t,
$$

where $\Phi$ is defined in (5.12), with $\rho=G(u)$ and $p \in W(0, T)$ being the associated adjoint state.

Proof. Let $z$ be the solution of the state equation linearized at $(\rho, u)$. Then one has

$$
\begin{aligned}
F^{\prime}(u) v & =\alpha_{Q} \int_{0}^{T} \int_{\Omega}\left(\rho-\rho_{Q}\right) z \mathrm{~d} x \mathrm{~d} t+\alpha_{\Omega} \int_{\Omega}\left(\rho(T)-\rho_{\Omega}\right) z(T) \mathrm{d} x+\sum_{i=1}^{n} \int_{0}^{T}\left(\gamma_{i} u_{i} v_{i}+\beta_{i} v_{i}\right) \mathrm{d} t \\
& =-\int_{0}^{T} \int_{\Omega} \rho(b \otimes v) \cdot \nabla p \mathrm{~d} x \mathrm{~d} t+\sum_{i=1}^{n} \int_{0}^{T}\left(\gamma_{i} u_{i} v_{i}+\beta_{i} v_{i}\right) \mathrm{d} t \\
& =\sum_{i=1}^{n} \int_{0}^{T}\left[-\int_{\Omega} \rho b_{i} \frac{\partial p}{\partial x_{i}} \mathrm{~d} x+\gamma_{i} u_{i}+\beta_{i}\right] v_{i} \mathrm{~d} t,
\end{aligned}
$$

where we used (5.11) in the second equality. This proves the result.

By means of the expression (5.13), we can reformulate the first order optimality condition of Proposition 4.5 as follows:

Corollary 5.5. If $\bar{u}$ is an $L^{\infty}$-local minimum for $(\mathrm{P})$, then

$$
\sum_{i=1}^{n} \int_{0}^{T} \bar{\Phi}_{i}(t)\left(u_{i}(t)-\bar{u}_{i}(t)\right) \mathrm{d} t \geq 0 \quad \text { for all } u \in \mathcal{U}_{\mathrm{ad}}
$$

where $\bar{\Phi}$ is the function given in (5.12) associated to $\bar{u}$. Consequently, we have

$$
\left\{\begin{array}{l}
\bar{\Phi}_{i}(t)>0 \Longrightarrow \bar{u}_{i}(t)=u_{i}^{\min }(t) \\
\bar{\Phi}_{i}(t)<0 \Longrightarrow \bar{u}_{i}(t)=u_{i}^{\max }(t) \\
u_{i}^{\min }(t)<\bar{u}_{i}(t)<u_{i}^{\max }(t) \Longrightarrow \bar{\Phi}_{i}(t)=0
\end{array}\right.
$$

a.e. on $[0, T]$ and for all $i=1, \ldots, n$. 


\section{SECOND ORDER ANALYSIS}

The optimal control problem is a non-convex one, hence first order necessary optimality conditions should be complemented by a second order analysis. Second order sufficient optimality conditions serve as important assumption for the numerical analysis. For instance, the stability of locally optimal solutions under a numerical approximation of the problem or the convergence analysis of numerical methods such as SQP or semismooth Newton techniques need second order sufficient optimality conditions as hypothesis. Though it is hardly possible to confirm them numerically, they are used as assumption for the analysis. This is similar to constraint qualifications in nonlinear optimization that can be verified only in exceptional cases but are indispensable for the analysis.

To establish second order optimality conditions, we will apply general results by Casas and Tröltzsch, ([16], Thms. 2.2 and 3.3). For this purpose, we have to verify that the conditions (C1)-(C3) below are satisfied for problem (P). More precisely, we will need $(\mathrm{C} 1)$ in the second order necessary condition of Theorem 6.6 below, while $(\mathrm{C} 1)-(\mathrm{C} 3)$ are used in the sufficient one of Theorem 6.13.

\subsection{Second order conditions for an optimization problem in Banach spaces}

We consider a Banach space $U_{\infty}$ and a Hilbert space $U_{2}$, endowed with the norms $|\cdot|_{\infty}$ and $|\cdot|_{2}$, respectively, and such that $U_{\infty}$ is continuously embedded in $U_{2}$. Let us introduce the abstract optimization problem

$$
\min _{u \in \mathcal{K}} \mathcal{J}(u)
$$

where $\mathcal{K} \subseteq U_{\infty}$ is a given nonempty convex set and $\mathcal{J}: \mathcal{A} \rightarrow \mathbb{R}$ is the objective function, defined and twice continuously differentiable in an open subset $\mathcal{A} \subset U_{\infty}$ that covers $\mathcal{K}$. We say that $\bar{u}$ is a $U_{\infty}$-local solution of $(\mathcal{P})$ if there exists $\varepsilon>0$ such that $\mathcal{J}(\bar{u}) \leq \mathcal{J}(u)$ holds for all $u \in \mathcal{K} \cap\left\{u \in U_{\infty}:|u-\bar{u}|_{\infty}<\varepsilon\right\}$.

If $\bar{u}$ is a $U_{\infty}$-local solution of $(\mathcal{P})$, then the following first order necessary condition is satisfied:

$$
\mathcal{J}^{\prime}(\bar{u})(u-\bar{u}) \geq 0 \quad \text { for all } u \in \mathcal{K}
$$

Let us fix $\bar{u}$ in $\mathcal{K}$. We consider the following conditions for problem $(\mathcal{P})$. All the notions of differentiability of $\mathcal{J}$ are to be understood in the sense of $U_{\infty}$.

(C1) The functional $\mathcal{J}$ is of class $C^{2}$ in $\mathcal{A}$. For every $u \in \mathcal{K}$, there exist continuous extensions

$$
\mathcal{J}^{\prime}(u) \in \mathcal{L}\left(U_{2} ; \mathbb{R}\right), \quad \mathcal{J}^{\prime \prime}(u) \in \mathcal{B}\left(U_{2} ; \mathbb{R}\right)
$$

of $\mathcal{J}^{\prime}(u)$ and $\mathcal{J}^{\prime \prime}(u)$, where $\mathcal{B}\left(U_{2} ; \mathbb{R}\right)$ denotes the Banach space of continuous bilinear real functionals on $U_{2} \times U_{2}$.

(C2) For any sequence $\left\{\left(u_{k}, v_{k}\right)\right\} \subset \mathcal{K} \times U_{2}$ with $u_{k} \rightarrow \bar{u}$ in $U_{2}$ and $v_{k} \rightarrow v$ weakly in $U_{2}$ as $k \rightarrow \infty$, there holds

$$
\mathcal{J}^{\prime}(\bar{u}) v=\lim _{k \rightarrow \infty} \mathcal{J}^{\prime}\left(u_{k}\right) v_{k}
$$

(C3) For any sequence defined as in (C2), the following two properties are satisfied for some $\Lambda>0$ : it holds

$$
\mathcal{J}^{\prime \prime}(\bar{u}) v^{2} \leq \liminf _{k \rightarrow \infty} \mathcal{J}^{\prime \prime}\left(u_{k}\right) v_{k}^{2},
$$

and, if $v=0$, then $\Lambda \liminf _{k \rightarrow \infty}\left|v_{k}\right|_{2}^{2} \leq \liminf _{k \rightarrow \infty} \mathcal{J}^{\prime \prime}\left(u_{k}\right) v_{k}^{2}$. 
For a fixed control $\bar{u} \in \mathcal{K}$, let us define the following sets

$$
\begin{aligned}
S(\bar{u}) & :=\left\{v \in U_{\infty}: v=\lambda(u-\bar{u}) \text { for some } \lambda>0 \text { and } u \in \mathcal{K}\right\} \\
C(\bar{u}) & :=\operatorname{cl}_{U_{2}}(S(\bar{u})) \cap\left\{v \in U_{2}: \mathcal{J}^{\prime}(\bar{u}) v=0\right\} \\
D(\bar{u}) & :=\left\{v \in S(\bar{u}): \mathcal{J}^{\prime}(\bar{u}) v=0\right\}
\end{aligned}
$$

The set $S(\bar{u})$ is called cone of feasible directions, while $C(\bar{u})$ is the critical cone.

We first state the general Theorems 2.2 and 2.3 from [16], that we will apply to obtain the second order necessary and sufficient optimality conditions for the control of our Fokker-Planck equation in Theorems 6.6 and 6.13 below.

Theorem 6.1 (Casas-Tröltzsch [16]). Let $\bar{u}$ be a $U_{\infty}$-local solution for $(\mathcal{P})$. Assume that $(\mathrm{C} 1)$ and the regularity condition $C(\bar{u})=\operatorname{cl}_{U_{2}} D(\bar{u})$ hold. Then

$$
\mathcal{J}^{\prime \prime}(\bar{u}) v^{2} \geq 0 \quad \text { for all } v \in C(\bar{u})
$$

Theorem 6.2 (Casas-Tröltzsch [16]). Suppose that (C1)-(C3) are fulfilled for problem (P). Let $\bar{u} \in \mathcal{K}$ satisfy the first order necessary condition (6.1) along with

$$
\mathcal{J}^{\prime \prime}(\bar{u}) v^{2}>0 \quad \text { for all } v \in C(\bar{u}) \backslash\{0\}
$$

Then, there exist $\varepsilon>0$ and $\delta>0$ such that

$$
\mathcal{J}(\bar{u})+\frac{\delta}{2}|u-\bar{u}|_{2}^{2} \leq \mathcal{J}(u) \quad \text { for all } u \in \mathcal{K} \cap B_{2, \varepsilon}(\bar{u})
$$

where $B_{2, \varepsilon}(\bar{u})$ is the open ball in $U_{2}$ of radius $\varepsilon$ and centered in the origin.

Remark 6.3. The two theorems above were formulated for problems where the so-called two-norm discrepancy occurs. This means first that the objective functional $\mathcal{J}$ is not of class $C^{2}$ in $U_{2}$, while it is $C^{2}$ in $U_{\infty}$. Second, it includes that the coercivity $\mathcal{J}^{\prime \prime}(\bar{u}) v^{2} \geq \delta|v|_{\infty}^{2}$ cannot be shown for the $U_{\infty}$-norm for any $\delta>0$, while it can possibly be fulfilled with the norm $|\cdot|_{2}$ of $U_{2}$. We refer e.g. to Ioffe, [28]. In our optimal control problem, the two-norm discrepancy does not occur, since the reduced objective functional $F$ belongs to the class $C^{\infty}$ in $U_{2}$. Therefore, we can work with $U_{\infty}=U_{2}=L^{2}\left(0, T ; \mathbb{R}^{n}\right)$.

In the remainder of this paper, we will confirm the three conditions (C1)-(C3) for our optimal control problem $(\mathrm{P})$. For this purpose, we consider

$$
\mathcal{A}=U_{\infty}=U_{2}:=L^{2}\left(0, T ; \mathbb{R}^{n}\right), \quad \mathcal{J}:=F, \quad \mathcal{K}:=\mathcal{U}_{\mathrm{ad}} .
$$

\subsection{Second derivative of the reduced cost functional}

Let us compute the second derivative $G^{\prime \prime}(u)\left[v_{1}, v_{2}\right]$ of the control-to-state operator, for $u \in L^{\infty}\left(0, T ; \mathbb{R}^{n}\right)$ and $v_{1}, v_{2} \in L^{2}\left(0, T ; \mathbb{R}^{n}\right)$. Notice that the differentiability of $G$ has already been proven in Corollary 4.2 . We have

$$
\partial_{t} G(u)-\nu \Delta G(u)-\operatorname{div}(B[u] G(u))=0
$$


subject to associated initial and boundary conditions. Then, differentiating with respect to $v_{1} \in L^{2}\left(0, T ; \mathbb{R}^{n}\right)$, we obtain

$$
\partial_{t}\left(G^{\prime}(u) v_{1}\right)-\nu \Delta\left(G^{\prime}(u) v_{1}\right)-\operatorname{div}\left(b \otimes v_{1} G(u)\right)-\operatorname{div}\left(B[u] G^{\prime}(u) v_{1}\right)=0 .
$$

Another differentiation with respect to $v_{2} \in L^{2}\left(0, T ; \mathbb{R}^{n}\right)$ yields

$$
\partial_{t}\left(G^{\prime \prime}(u)\left[v_{1}, v_{2}\right]\right)-\nu \Delta\left(G^{\prime \prime}(u)\left[v_{1}, v_{2}\right]\right)-\operatorname{div}\left(b \otimes v_{1} G^{\prime}(u) v_{2}\right)-\operatorname{div}\left(b \otimes v_{2} G^{\prime}(u) v_{1}\right)-\operatorname{div}\left(B[u] G^{\prime \prime}(u)\left[v_{1}, v_{2}\right]\right)=0 .
$$

Setting $z_{i}:=G^{\prime}(u) v_{i}$, for $i=1,2$, and $w:=G^{\prime \prime}(u)\left[v_{1}, v_{2}\right]$ for the unknown second-order derivative, we get the following equation for $w$, written in strong form:

$$
\begin{aligned}
w_{t}-\nu \Delta w-\operatorname{div}(B[u] w) & =\operatorname{div}\left(z_{1} b \otimes v_{2}+z_{2} b \otimes v_{1}\right), \\
(\nu \nabla w+w B[u]) \cdot n & =-\left(z_{1} b \otimes v_{2}+z_{2} b \otimes v_{1}\right) \cdot n, \\
w(0) & =0 .
\end{aligned}
$$

The boundary condition is also obtained by implicit differentiation. Notice that $v_{1}$ and $v_{2}$ depend only on $t$. Moreover, we have $b \in L^{\infty}\left(\Omega ; \mathbb{R}^{n}\right)$ and $z_{i} \in W(0, T)$, for $i=1$, 2. In particular, $z_{i}$ belongs to $C\left([0, T] ; L^{2}(\Omega)\right)$, for $i=1,2$. Therefore, $z_{1} b \otimes v_{2}+z_{2} b \otimes v_{1}$ are in $L^{2}\left(Q ; \mathbb{R}^{n}\right)$. The homogeneous initial condition follows obviously by differentiating the equation $G(u)(\cdot, 0)=\rho_{0}(\cdot)$ twice with respect to $u$. The associated weak formulation is

$$
\begin{aligned}
\frac{\mathrm{d}}{\mathrm{d} t}(w, \varphi)+a[u](w, \varphi) & =\left(\mathrm{d}\left[z_{1} b \otimes v_{2}\right]+\mathrm{d}\left[z_{2} b \otimes v_{1}\right]\right)(\varphi) \quad \text { on } \mathcal{D}^{\prime}(0, T) \text { for all } \varphi \in H^{1}(\Omega), \\
w(0) & =0 \quad \text { in } L^{2}(\Omega) .
\end{aligned}
$$

Proposition 6.4. Let $u \in L^{\infty}\left(0, T ; \mathbb{R}^{n}\right)$ be given and $v_{1}, v_{2} \in L^{2}\left(0, T ; \mathbb{R}^{n}\right)$ be control increments with associated linearized states $z_{1}, z_{2}$, respectively. Then the second order derivative of the reduced cost functional $F$ at $u$ in the direction pair $\left(v_{1}, v_{2}\right)$ is given by

$F^{\prime \prime}(u)\left[v_{1}, v_{2}\right]=\int_{0}^{T} \int_{\Omega}\left(\alpha_{Q} z_{1} z_{2}-\nabla p \cdot\left(z_{2} b \otimes v_{1}+z_{1} b \otimes v_{2}\right)\right) \mathrm{d} x \mathrm{~d} t+\int_{0}^{T} \sum_{i=1}^{n} \gamma_{i} v_{1, i} v_{2, i} \mathrm{~d} t+\alpha_{\Omega} \int_{\Omega} z_{1}(T) z_{2}(T) \mathrm{d} x$,

where $p \in W(0, T)$ is the adjoint state associated with $u$.

Proof. The second order derivative of $F$ is computed by the chain rule. One has

$$
\begin{aligned}
F^{\prime}(u) v_{1}= & \alpha_{Q}\left(G(u)-\rho_{Q}, G^{\prime}(u) v_{1}\right)_{L^{2}(Q)} \\
& +\alpha_{\Omega}\left((G(u))(T)-\rho_{\Omega},\left(G^{\prime}(u) v_{1}\right)(T)\right)_{L^{2}(\Omega)}+\sum_{i=1}^{n}\left(\gamma_{i}\left(u_{i}, v_{1, i}\right)_{L^{2}(0, T)}+\beta_{i} \int_{0}^{T} v_{1, i} \mathrm{~d} t\right) .
\end{aligned}
$$


Then, differentiating this expression w.r.t. $v_{2}$ yields

$$
\begin{aligned}
F^{\prime \prime}(u)\left[v_{1}, v_{2}\right]= & \alpha_{Q}\left(G^{\prime}(u) v_{2}, G^{\prime}(u) v_{1}\right)_{L^{2}(Q)}+\alpha_{Q}\left(G(u)-\rho_{Q}, G^{\prime \prime}(u)\left[v_{1}, v_{2}\right]\right)_{L^{2}(Q)} \\
& +\alpha_{\Omega}\left(\left(G^{\prime}(u) v_{2}\right)(T),\left(G^{\prime}(u) v_{1}\right)(T)\right)_{L^{2}(\Omega)} \\
& +\alpha_{\Omega}\left((G(u))(T)-\rho_{\Omega},\left(G^{\prime \prime}(u)\left[v_{1}, v_{2}\right]\right)(T)\right)_{L^{2}(\Omega)}+\sum_{i=1}^{n} \gamma_{i}\left(v_{2, i}, v_{1, i}\right)_{L^{2}(0, T)} \\
= & \alpha_{Q}\left(z_{1}, z_{2}\right)_{L^{2}(Q)}+\alpha_{Q}\left(G(u)-\rho_{Q}, w\right)_{L^{2}(Q)}+\alpha_{\Omega}\left(z_{1}(T), z_{2}(T)\right)_{L^{2}(\Omega)} \\
& +\alpha_{\Omega}(p(T), w(T))_{L^{2}(\Omega)}+\sum_{i=1}^{n} \gamma_{i}\left(v_{1, i}, v_{2, i}\right)_{L^{2}(0, T)}
\end{aligned}
$$

where we used that $z_{i}=G^{\prime}(u) v_{i}$, for $i=1,2$, and $w=G^{\prime \prime}(u)\left[v_{1}, v_{2}\right]$. Moreover, we invoked the terminal condition for $p$ from the adjoint equation (5.1). This form of $F^{\prime \prime}$ includes the solution $w$ that implicitly depends on the increments $v_{1}, v_{2}$ via the partial differential equation (6.8). Now we proceed in the same way that we used to show equation (5.11). We insert $p$ as test function in equation (6.8) and $w$ as test function in the adjoint equation (5.4). After some integration by parts, we arrive at the relation

$$
\alpha_{Q}\left(G(u)-\rho_{Q}, w\right)_{L^{2}(Q)}+\alpha_{\Omega}(p(T), w(T))_{L^{2}(\Omega)}=-\left(\nabla p, b \otimes v_{1} z_{2}+b \otimes v_{2} z_{1}\right)_{L^{2}(Q)}
$$

that yields (5.11). Inserting this result in (6.11), we verify the claim.

We will state our second order optimality conditions in terms of the quadratic form

$$
F^{\prime \prime}(u) v^{2}=\iint_{Q}\left[\alpha_{Q} z^{2}-2 \nabla p \cdot(z b \otimes v)\right] \mathrm{d} x \mathrm{~d} t+\sum_{i=1}^{n} \gamma_{i} \int_{0}^{T} v_{i}^{2} \mathrm{~d} t+\alpha_{\Omega} \int_{\Omega} z(T)^{2} \mathrm{~d} x
$$

that is obtained from the general form (6.10) of $F^{\prime \prime}$ by the choice $v_{1}=v_{2}=v \in L^{2}\left(0, T ; \mathbb{R}^{n}\right)$. For all $u \in U_{\infty}=$ $L^{\infty}\left(0, T ; \mathbb{R}^{n}\right)$, the quadratic form is defined and continuous in $U_{2}=L^{2}\left(0, T ; \mathbb{R}^{n}\right)$.

\subsection{Critical directions}

For the sets defined in (6.5), the following characterization is easily obtained.

Proposition 6.5 (Characterization of the critical cone).

$$
C(\bar{u})=\left\{\begin{array}{c}
v \in L^{2}\left(0, T ; \mathbb{R}^{n}\right): \text { a.e. on }[0, T] \text { and for } i=1, \ldots, n, \\
v_{i}\left\{\begin{array}{ll}
\geq 0 & \text { if } \bar{u}_{i}=u_{i}^{\min } \\
\leq 0 & \text { if } \bar{u}_{i}=u_{i}^{\max } \\
=0 & \text { if } \bar{\Phi}_{i} \neq 0
\end{array}\right\},
\end{array}\right.
$$

where $\bar{\Phi}$ was defined in (5.12).

Proof. We follow essentially the lines of the proof in ([16], p. 273). Let us use $K(\bar{u})$ to denote the set on the r.h.s. of (6.13).

First take $v \in C(\bar{u})$. Then $F^{\prime}(\bar{u}) v=0$ and $v$ is in $C(\bar{u})$ so by definition $v \in C(\bar{u})$, we used this below, we do not deduce it there exists $\left\{v_{k}\right\} \subseteq S(\bar{u})$ such that $v_{k} \rightarrow v$ in $L^{2}\left(0, T ; \mathbb{R}^{n}\right)$. By definition of $S(\bar{u})$ one has, necessarily, that $v_{k, i} \geq 0$ if $\bar{u}_{i}=u_{i}^{\min }$ and $v_{k, i} \leq 0$ if $\bar{u}_{i}=u_{i}^{\max }$, a.e. on $[0, T]$ and for every $i=1, \ldots, n$. Clearly, 
this property is preserved for the limit in $L^{2}\left(0, T ; \mathbb{R}^{n}\right)$, so that it also holds for $v$. From this fact, due to the expression of $F^{\prime}$ given in (5.13) and the first order necessary condition of (5.16), we deduce that

$$
0=F^{\prime}(\bar{u}) v=\sum_{i=1}^{n} \int_{0}^{T} \bar{\Phi}_{i}(t) v_{i}(t) \mathrm{d} t=\sum_{i=1}^{n} \int_{0}^{T}\left|\bar{\Phi}_{i}(t) v_{i}(t)\right| \mathrm{d} t
$$

which implies that $v_{i}=0$ if $\bar{\Phi}_{i} \neq 0$. This proves that $C(\bar{u}) \subseteq K(\bar{u})$.

In order to prove the converse inclusion, take $v \in K(\bar{u})$, and define, for each positive integer $k$ and for each $i=1, \ldots, n$,

$$
v_{k, i}(t):=\left\{\begin{array}{l}
0 \quad \text { if } u_{i}^{\min }(t)<\bar{u}_{i}(t)<u_{i}^{\min }(t)+\frac{1}{k} \text { or } u_{i}^{\max }(t)-\frac{1}{k}<\bar{u}_{i}(t)<u_{i}^{\max }(t), \\
\mathbb{P}_{[-k, k]}\left(v_{i}(t)\right) \quad \text { otherwise, }
\end{array}\right.
$$

where $\mathbb{P}_{[-k, k]}$ denotes the pointwise projection onto the interval $[-k, k]$. Thus $v_{k} \in L^{\infty}\left(0, T ; \mathbb{R}^{n}\right), v_{k} \rightarrow v$ in $L^{2}\left(0, T ; \mathbb{R}^{n}\right)$ and it easily follows that $u_{k}:=\bar{u}+\lambda_{k} v_{k}$ belongs to $\mathcal{U}_{\text {ad }}$ for $\lambda_{k}:=\frac{1}{k^{2}}$; hence $v_{k} \in S(\bar{u})$. Thus $K(\bar{u}) \subseteq \mathrm{cl}_{L^{2}}(S(\bar{u}))$. Finally, we observe that by definition of $C(\bar{u})$ and the expression (5.13) of $F^{\prime}$, one has that $F^{\prime}(\bar{u}) v=0$. This shows that $v \in C(\bar{u})$ and concludes the proof.

\subsection{Second order necessary optimality conditions}

In view of the second order continuous differentiability of $F$ in $L^{2}\left(0, T ; \mathbb{R}^{n}\right)$, the conditions $(\mathrm{C} 1)$ and $(\mathrm{C} 2)$ are trivially satisfied for our control problem. Therefore, we can directly apply the general Theorem 6.1 and set up second order necessary conditions for the control of our Fokker-Planck equation.

Theorem 6.6 (Second order necessary optimality condition). Let $\bar{u}$ be an $L^{\infty}$-local solution for (P). Then

$$
F^{\prime \prime}(\bar{u}) v^{2} \geq 0 \quad \text { for all } v \in C(\bar{u}) .
$$

Proof. In view of Theorem 6.1, it only remains to prove that $C(\bar{u})=\operatorname{cl}_{L^{2}} D(\bar{u})$. The inclusion $C(\bar{u}) \supseteq \operatorname{cl}_{L^{2}} D(\bar{u})$ follows easily from the expression of $F^{\prime}$ given in (5.13), since $\bar{\Phi}$ belongs to $L^{2}\left(0, T ; \mathbb{R}^{n}\right)$. Let us show the converse inclusion, $C(\bar{u}) \subseteq \operatorname{cl}_{L^{2}} D(\bar{u})$. Take $v \in C(\bar{u})$, and define $v_{k}$, for each positive integer $k$, as done in (6.14). Hence, we have $v_{k} \in S(\bar{u})$. By definition, we know that $v_{k} \rightarrow v$ strongly in $L^{2}\left(0, T ; \mathbb{R}^{n}\right)$. Moreover, since $\left|v_{k, i}(t)\right| \leq\left|v_{i}(t)\right|$ a.e. on $[0, T]$ for every $i=1, \ldots, n$, we have in addition that $\left|\bar{\Phi}_{i}(t) v_{k, i}(t)\right| \leq\left|\bar{\Phi}_{i}(t) v_{i}(t)\right|=0$ a.e. on $[0, T]$. This implies $F^{\prime}(\bar{u}) v_{k}=0$. Consequently, $v_{k} \in D(\bar{u})$ holds for every $k$ and, hence, $v \in \operatorname{cl}_{L^{2}} D(\bar{u})$. This yields the desired inclusion.

Finally, the necessary condition (6.15) follows from Theorem 6.1.

\subsection{Second order sufficient conditions}

To apply the second order sufficient condition of the abstract Theorem 6.2 , we impose the following two additional assumptions, that are assumed to hold throughout the remainder of the article. In the case without terminal observation, i.e. for $\alpha_{\Omega}=0$, they can be simplified.

Assumption 6.7. The function $b$ belongs to $W^{1, \infty}\left(\Omega ; \mathbb{R}^{n}\right)$ and, for a.a. $x \in \Gamma$, it holds

$$
(b(x) \otimes u) \cdot n(x)=0 \quad \text { for all } u \in \mathbb{R}^{n} .
$$

Remark 6.8. Assumption 6.7 above is fulfilled in particular, if $b \in W_{0}^{1, \infty}\left(\Omega ; \mathbb{R}^{n}\right)$. Moreover, it holds if $b(x)$. $n(x)=0$ a.e. on $\Gamma$ and the control is of the form $u(t)=\mathfrak{u}(t)(1, \ldots, 1) \in \mathbb{R}^{n}$, with $\mathfrak{u}$ being a scalar function. 
Assumption 6.9 (Requirements for higher regularity).

(i) The function $c$ has a potential $-V \in W^{2, \infty}(\Omega)$ so that

$$
c=\nabla V
$$

or $c$ belongs to $W^{1, \infty}\left(\Omega, \mathbb{R}^{n}\right)$ and satisfies

$$
c(x) \cdot n(x)=0 \quad \text { a.e. on } \Gamma,
$$

(ii) the initial and the desired distributions $\rho_{0}$ and $\rho_{\Omega}$ belong to $H^{1}(\Omega)$,

(iii) and

$$
\min _{i=1, \ldots, n} \gamma_{i}>0
$$

Remark 6.10. The assumption (6.16) that $c$ has a potential was imposed by Breiten et al. [13] to gain higher regularity of $\rho$.

For smooth domains and a real-valued control, the next result follows from Breiten, Kunisch and Pfeiffer ([13], Prop. 6.1), who proved $W\left(0, T ; H^{2}(\Omega), L^{2}(\Omega)\right)$-regularity of $\rho$ by a Galerkin technique. We extend the $C\left([0, T] ; H^{1}(\Omega)\right)$-regularity of $\rho$ to bounded Lipschitz domains and to our setting of a vector-valued control.

Theorem 6.11. If Assumptions 6.7 and 6.9 are fulfilled, then, given any control $u \in L^{\infty}\left(0, T ; \mathbb{R}^{n}\right)$, the unique weak solution $\rho$ of the Fokker-Planck equation (2.1), (2.7) belongs to $C\left([0, T] ; H^{1}(\Omega)\right)$.

The proof can be found in the Appendix A.

To apply the abstract Theorem 6.2, we are going to verify the conditions (C1)-(C3). As we have mentioned at the beginning of the preceding subsection, (C1)-(C2) are trivially satisfied. To confirm (C3), we simplify this condition in the following way. Since $F$ is of class $\mathcal{C}^{\infty}$ in $L^{2}\left(0, T ; \mathbb{R}^{n}\right)$, the second derivative $F^{\prime \prime}$ is locally Lipschitz, hence for all $u_{1}, u_{2}$ in an $L^{2}$-neighborhood of $\bar{u}$, we have

$$
\left|\left(F^{\prime \prime}\left(u_{1}\right)-F^{\prime \prime}\left(u_{2}\right)\right)\left[v_{1}, v_{2}\right]\right| \leq c\left\|u_{1}-u_{2}\right\|_{2}\left\|v_{1}\right\|_{2}\left\|v_{2}\right\|_{2} \quad \text { for all } v_{1}, v_{2} \in L^{2}\left(0, T ; \mathbb{R}^{n}\right),
$$

with some constant $c>0$. Therefore, for the sequences $\left\{u_{k}\right\}$ and $\left\{v_{k}\right\}$ defined in condition (C3) it follows that

$$
\liminf _{k \rightarrow \infty} F^{\prime \prime}\left(u_{k}\right) v_{k}^{2}=\liminf _{k \rightarrow \infty} F^{\prime \prime}(\bar{u}) v_{k}^{2}+\lim _{k \rightarrow \infty}\left(F^{\prime \prime}\left(u_{k}\right)-F^{\prime \prime}(\bar{u})\right) v_{k}^{2}=\liminf _{k \rightarrow \infty} F^{\prime \prime}(\bar{u}) v_{k}^{2} .
$$

In this way, it suffices to confirm conditions (6.3) and (6.4) of (C3) at the fixed control $\bar{u}$ (instead of $u_{k}$ ). We thank one of the referees for suggesting this essential simplification. A version of the manuscript with proofs that hold for costs that are merely twice continuously differentiable in $L^{\infty}\left(0, T ; \mathbb{R}^{m}\right)$ is available at [9].

Proposition 6.12. Under Assumptions 6.7 and 6.9, problem (P) satisfies condition (C3). If $\alpha_{\Omega}=0$, then the Assumptions 6.7 and 6.9 can be substituted by $\min _{i=1, \ldots, n} \gamma_{i}>0$.

Proof. In view of our comments above, it suffices to verify the condition (6.18). To this end, we consider a sequence $\left\{v_{k}\right\} \subset L^{2}\left(0, T ; \mathbb{R}^{n}\right)$ with $v_{k} \rightarrow v$ weakly in $L^{2}\left(0, T ; \mathbb{R}^{n}\right)$ as in $(\mathrm{C} 3)$. For each $k$, let $z_{k}$ be the linearized state associated to the control $\bar{u}$ in the direction $v_{k}$. We will proceed in several steps.

1) Preparations. The linearized states $z_{k}$ are defined by $z_{k}=G^{\prime}(\bar{u}) v_{k}$ and the operator $G^{\prime}(\bar{u})$ is linear and continuous from $L^{2}\left(0, T ; \mathbb{R}^{n}\right)$ to $W(0, T)$, hence weakly continuous. Therefore, the sequence $\left\{z_{k}\right\}$ converges weakly in $W(0, T)$ to the function $z \in W(0, T)$ given by $z=G^{\prime}(\bar{u}) v$. We have shown that $z$ is the linearized state associated with $\bar{u}$ in the direction of the weak limit $v$. 
In order to show (6.18), we select a subsequence $\left\{v_{k_{j}}\right\}$ such that

$$
\liminf _{k \rightarrow \infty} F^{\prime \prime}(\bar{u}) v_{k}^{2}=\lim _{j \rightarrow \infty} F^{\prime \prime}(\bar{u}) v_{k_{j}}^{2}
$$

The corresponding subsequence $\left\{z_{k_{j}}\right\}$ converges weakly to $z$ in $W(0, T)$ and hence, in view of Aubin-Lions' Lemma [11], it contains a subsequence $\left\{z_{k_{j_{\ell}}}\right\}$ that converges strongly to $z$, i.e.

$$
z_{k_{j_{\ell}}} \rightarrow z \text { in } L^{2}(Q) .
$$

In order to simplify the notation, let us use the subindex $k$ for this subsequence. One has

$$
F^{\prime \prime}(\bar{u}) v_{k}^{2}=\iint_{Q}\left[z_{k}^{2}-2 \nabla \bar{p} \cdot\left(z_{k} b \otimes v_{k}\right)\right] \mathrm{d} x \mathrm{~d} t+\sum_{i=1}^{n} \gamma_{i} \int_{0}^{T} v_{k, i}^{2} \mathrm{~d} t+\int_{\Omega} z_{k}(T)^{2} \mathrm{~d} x,
$$

for $z_{k}$ as given above and $\bar{p}$ being the adjoint state associated with $\bar{u}$.

Thanks to Theorem 6.11 , the optimal state $\bar{\rho}$ associated with $\bar{u}$ belongs to $C\left([0, T] ; H^{1}(\Omega)\right)$. Moreover, we obtain $\bar{p} \in C\left([0, T] ; H^{1}(\Omega)\right)$. This can be seen as follows: from the adjoint equation (5.1), we get

$$
\begin{aligned}
-\partial_{t} \bar{p}-\nu \Delta \bar{p} & =\alpha_{Q}\left(\bar{\rho}-\rho_{Q}\right)-B[\bar{u}] \cdot \nabla \bar{p} \quad \text { in } Q \\
\bar{p}(T) & =\alpha_{\Omega}\left(\bar{\rho}(T)-\rho_{\Omega}\right) \quad \text { in } \Omega \\
\partial_{n} \bar{p} & =0 \quad \text { on } \Sigma .
\end{aligned}
$$

Since $\bar{u} \in L^{\infty}\left(0, T ; \mathbb{R}^{n}\right)$, the r.h.s. $\alpha_{Q}\left(\bar{\rho}-\rho_{Q}\right)-B[\bar{u}] \cdot \nabla \bar{p}$ belongs to $L^{2}(Q)$. Moreover, by Assumption 6.9 , (ii), and by the regularity $\bar{\rho} \in C\left([0, T] ; H^{1}(\Omega)\right), \alpha_{\Omega}\left(\bar{\rho}(T)-\rho_{\Omega}\right)$ is in $H^{1}(\Omega)$. We can apply the regularity-Lemma A.1 and get that $\bar{p}$ is contained in $C\left([0, T] ; H^{1}(\Omega)\right)$. If $\alpha_{\Omega}=0$, then the state $\bar{\rho}$ does not appear in $(6.21)$, hence we do not need the conditions on $b$ and $c$ of Assumptions 6.7 and 6.9 and require only the condition $\min _{i=1, \ldots, n} \gamma_{i}>0$.

2) Proof of (6.18). We are now ready to verify (6.18). Consider the expression of $F^{\prime \prime}(\bar{u}) v_{k}^{2}$ given in (6.20). From (6.19), we know that $z_{k} \rightarrow z$ (strongly) in $L^{2}(Q)$. Moreover, we have $v_{k} \rightarrow v$ in $L^{2}\left(0, T ; \mathbb{R}^{n}\right)$. Therefore, the product $z_{k} b \otimes v_{k}$ converges weakly in $L^{1}\left(0, T ; L^{2}(\Omega)^{n}\right)$ to $z b \otimes v$. Thanks to the higher regularity $\bar{p} \in$ $C\left([0, T] ; H^{1}(\Omega)\right)$, it holds $\nabla \bar{p} \in C\left([0, T] ; L^{2}(\Omega)^{n}\right)$. For the first integral in (6.20), these properties imply that

$$
\iint_{Q}\left[z_{k}^{2}-2 \nabla \bar{p} \cdot\left(z_{k} b \otimes v_{k}\right)\right] \mathrm{d} x \mathrm{~d} t \rightarrow \iint_{Q}\left[z^{2}-2 \nabla \bar{p} \cdot(z b \otimes v)\right] \mathrm{d} x \mathrm{~d} t, \quad k \rightarrow \infty .
$$

It remains to pass to the limit in the other terms of $F^{\prime \prime}(\bar{u}) v_{k}^{2}$. Due to the weak convergence $v_{k} \rightarrow v$ in $L^{2}\left(0, T ; \mathbb{R}^{n}\right)$, we get

$$
\sum_{i=1}^{n} \gamma_{i} \int_{0}^{T} v_{i}^{2} \mathrm{~d} t \leq \liminf _{k \rightarrow \infty} \sum_{i=1}^{n} \gamma_{i} \int_{0}^{T} v_{k, i}^{2} \mathrm{~d} t
$$

Since the mapping $z \mapsto z(\cdot, T)$ is linear and continuous from $W(0, T)$ to $L^{2}(\Omega)$, it is also weakly lower semicontinuous and, therefore,

$$
\int_{\Omega} z(T)^{2} \mathrm{~d} x \leq \liminf _{k \rightarrow \infty} \int_{\Omega} z_{k}(T)^{2} \mathrm{~d} x
$$

Adding the expressions (6.22)-(6.24), we arrive at (6.3). 
3) Proof of (6.4). It remains to show (6.4). To this end, we assume that $v=0$, this is $v_{k} \rightarrow 0$ in $L^{2}\left(0, T ; \mathbb{R}^{n}\right)$. Thus, the corresponding limit $z$ of $\left\{z_{k}\right\}$ is also zero. Again, we are justified to consider (6.4) for the stationary sequence $u_{k}=\bar{u}$ for all $k \in \mathbb{N}$. We get

$$
\liminf _{k \rightarrow \infty} F^{\prime \prime}(\bar{u}) v_{k}^{2}=\liminf _{k \rightarrow \infty}\left[\sum_{i=1}^{n} \gamma_{i} \int_{0}^{T} v_{k, i}^{2} \mathrm{~d} t+\int_{\Omega} z_{k}(T)^{2} \mathrm{~d} x\right] \geq\left(\min _{i=1, \ldots, n} \gamma_{i}\right) \liminf _{k \rightarrow \infty}\left\|v_{k}\right\|_{2}^{2}
$$

This yields (6.4) for $\Lambda:=\min _{i=1, \ldots, n} \gamma_{i}>0$, and then the proof is concluded.

Now we come to the main result of our paper.

Theorem 6.13 (Second order sufficient condition). Let $\bar{u} \in \mathcal{U}_{\mathrm{ad}}$ be such that the first order condition (4.9) is satisfied, and

$$
F^{\prime \prime}(\bar{u}) v^{2}>0, \quad \text { for all } v \in C(\bar{u}) \backslash\{0\} .
$$

Under the Assumptions 6.7 and 6.9 that can be simplified by $\min _{i=1, \ldots, n} \gamma_{i}>0$, if $\alpha_{\Omega}=0$, there exist ....

$$
J(\bar{u})+\frac{\delta}{2}\|u-\bar{u}\|_{2}^{2} \leq F(u), \quad \text { for all } u \in \mathcal{U}_{\mathrm{ad}} \cap B_{\varepsilon}^{2}(\bar{u}),
$$

holds, hence $\bar{u}$ is locally optimal in the sense of $L^{2}\left(0, T ; \mathbb{R}^{n}\right)$.

Proof. The result follows from the application of Theorem 6.2 to our optimal control problem (P). The associated assumptions are fulfilled in view of the second order continuous differentiability of $F$ in $L^{2}\left(0, T ; \mathbb{R}^{n}\right)$ and Proposition 6.12.

Remark 6.14. We should mention that also ([16], Cor. 2.6) on local uniqueness of $\bar{u}$ and ([16], Thm. 2.7) regarding an alternative critical cone can be directly transferred to our optimal control problem, since the required assumptions $(\mathrm{C} 1)-(\mathrm{C} 3)$ are satisfied.

\section{APPENDix A.}

In this section, we prove the regularity result of Theorem 6.11. First, we show that under the Assumptions 6.7 and 6.9 the state equation for $\rho$ can be transformed to a linear heat equation with homogeneous Neumann condition and right-hand side in $L^{2}(Q)$. This is the key for higher regularity of $\rho$.

To see this, we begin with the case where Assumption 6.7 and condition (6.17) are fulfilled. Then, for any control function $u \in L^{\infty}\left(0, T ; \mathbb{R}^{n}\right), \operatorname{div}(\rho(c+b \otimes u))=\operatorname{div}(\rho B[u]) \in L^{2}(Q)$ and

$$
-\int_{\Omega} \rho(c+b \otimes u) \cdot \nabla \varphi \mathrm{d} x=\int_{\Omega} \operatorname{div}(\rho(c+b \otimes u)) \varphi \mathrm{d} x \quad \text { for all } \varphi \in H^{1}(\Omega), u \in \mathbb{R}^{n}
$$

since $\rho(c+b \otimes u) \cdot n=\rho B[u] \cdot n=0$ on $\Gamma$. Therefore, the state equation (2.1), (2.7) reduces to the linear heat equation

$$
\begin{aligned}
\partial_{t} \rho-\nu \Delta \rho & =\hat{f}, \\
\rho(0) & =\rho_{0}, \\
\nabla \rho \cdot n & =0,
\end{aligned}
$$


where

$$
\hat{f}:=\operatorname{div}(\rho B[u]) \in L^{2}(Q) .
$$

Next, we confirm the reduction to an equation of the form (A.1), if $c$ does not satisfy (6.17) but fulfils (6.16). In this case, we follow an idea of [13], proof of Proposition 2.1, and apply the transformation $w=\exp (V / \nu) \rho$. Notice that $w$ enjoys the same regularity as $\rho$, i.e. $w \in W(0, T)$. We find

$$
\nabla \rho=-\frac{1}{\nu} \exp (-V / \nu) \nabla V w+\exp (-V / \nu) \nabla w=\exp (-V / \nu)\left(-\frac{1}{\nu} c w+\nabla w\right) .
$$

Inserting this in the boundary condition of the state equation, we arrive at

$$
0=\nu \nabla \rho \cdot n+\rho B[u] \cdot n=\exp (-V / \nu)(-c w+\nu \nabla w) \cdot n+\exp (-V / \nu) w(c+b \otimes u) \cdot n=\nu \exp (-V / \nu) \nabla w \cdot n
$$

that holds true if, and only if, $\nabla w \cdot n=0$ on $\Gamma$. Therefore, $w$ satisfies homogeneous Neumann boundary conditions. Moreover, inserting the transformation of $\rho$ in the state equation, we obtain, after some computations by the product rule applied to $\operatorname{div} \nabla(\exp (-V / \nu) w)$ that $w$ satisfies the linear heat equation (A.1) with right-hand side

$$
\hat{f}=-c \cdot \nabla w-\frac{1}{\nu} w c \cdot b \otimes u+\operatorname{div}(w b \otimes u) .
$$

Since $w \in W(0, T)$ and $\nabla w \cdot n=0$ on $\Gamma$, all summands above belong to $L^{2}(Q)$.

Next, we provide a regularity result for equation (A.1) that can be found, for homogeneous Dirichlet boundary conditions and a smooth boundary $\Gamma$, in Evans [22], Theorem 5, page 360 or Ladyzhenskaya et al. [30]. For the convenience of the reader, we will prove the part on $C\left([0, T] ; H^{1}(\Omega)\right)$-regularity in Lipschitz domains. It is difficult to find an associated reference, although the result is known. The proof was communicated to us by Joachim Rehberg (WIAS Berlin).

Lemma A.1. If $\Omega$ is a bounded Lipschitz domain, $\hat{f}$ is an arbitrary function of $L^{2}(Q)$ and $\rho_{0}$ belongs to $H^{1}(\Omega)$, then the unique weak solution $\rho$ of the linear heat equation (A.1) enjoys the higher regularity $\rho \in$ $C\left([0, T] ; H^{1}(\Omega)\right)$. Moreover, the estimate

$$
\|\rho\|_{W(0, T)}+\|\rho\|_{C\left([0, T] ; H^{1}(\Omega)\right)} \leq C\left(\|\hat{f}\|_{L^{2}(Q)}+\left\|\rho_{0}\right\|_{H^{1}(\Omega)}\right)
$$

is satisfied with some $C>0$ not depending on $\hat{f}$ neither on $\rho_{0}$.

Proof. The Laplace operator $\Delta$ is generated in $L^{2}(\Omega)$ by the bilinear form

$$
(\psi, \varphi) \mapsto \int_{\Omega} \nabla \psi \cdot \nabla \varphi \mathrm{d} x
$$

defined on $H^{1}(\Omega) \times H^{1}(\Omega)$, (cf. [29], chpt. VI or [33], chpt. 1). This operator, induced in $L^{2}(\Omega)$, is non-positive and self-adjoint, hence it generates an analytic semigroup in $L^{2}(\Omega)$.

It is known that the negative of any linear operator in a Hilbert space that generates an analytic semigroup has maximal parabolic regularity. In our setting, this means the following: if $S$ is an arbitrary bounded real interval, for every $f \in L^{2}\left(S ; L^{2}(\Omega)\right)$ and each initial value $y_{0}$ from the real interpolation space $\left(L^{2}(\Omega), \operatorname{dom}_{L^{2}(\Omega)} \Delta\right)_{\frac{1}{2}, 2}$, there exists a unique $y \in W^{1,2}\left(S ; L^{2}(\Omega)\right) \cap L^{2}\left(S ; \operatorname{dom}_{L^{2}(\Omega)} \Delta\right)$ such that

$$
\partial_{t} y-\Delta y=f, \quad y(0)=y_{0} .
$$


For this existence result, we refer to [10], chapter 1.3, in particular to chapter 1.3.3. Moreover, one has (see e.g. [3], chpt. III.4.10)

$$
W^{1,2}\left(S ; L^{2}(\Omega)\right) \cap L^{2}\left(S, \operatorname{dom}_{L^{2}(\Omega)} \Delta\right) \hookrightarrow C\left(\bar{S} ;\left(L^{2}(\Omega), \operatorname{dom}_{L^{2}(\Omega)} \Delta\right)_{\frac{1}{2}, 2}\right) .
$$

The operator $I-\Delta$ is positive and self-adjoint with lower spectral bound 1 . Therefore, thanks to the spectral theory, its pure imaginary powers exist as bounded operators in $L^{2}(\Omega)$. In view of this, one has (see [37], chpt. 1.18.10)

$$
\begin{aligned}
\left(L^{2}(\Omega), \operatorname{dom}_{L^{2}(\Omega)} \Delta\right)_{\frac{1}{2}, 2} & =\left(L^{2}(\Omega), \operatorname{dom}_{L^{2}(\Omega)}(-\Delta+I)\right)_{\frac{1}{2}, 2} \\
& =\left[L^{2}(\Omega), \operatorname{dom}_{L^{2}(\Omega)}(-\Delta+I)\right]_{\frac{1}{2}}=\operatorname{dom}_{L^{2}(\Omega)}(-\Delta+I)^{\frac{1}{2}}
\end{aligned}
$$

Since $\Delta$ is self-adjoint, the space $\operatorname{dom}_{L^{2}(\Omega)}(-\Delta+I)^{\frac{1}{2}}$ is equal to its form-domain, i.e. $H^{1}(\Omega)$, [29, chpt. VI 2.6]. Therefore, (A.5) implies

$$
W^{1,2}\left(S ; L^{2}(\Omega)\right) \cap L^{2}\left(S, \operatorname{dom}_{L^{2}(\Omega)} \Delta\right) \hookrightarrow C\left(\bar{S} ; H^{1}(\Omega)\right) .
$$

Exactly the same arguments are true for $\nu \Delta$ instead of $\Delta$, except that the lower spectral bound is now estimated from below by $\nu$. These statements are valid in arbitrary domains $\Omega$. Moreover, the interval $S$ is allowed to be unbounded, if the smallest eigenvalue of $-\Delta$ under the given homogeneous boundary conditions is positive, e.g., in $H_{0}^{1}(\Omega)$. For the Neumann-Laplace operator, this is not the case.

The estimate (A.4) is shown as usual by considering a graph norm.

Proof of Theorem 6.11: We have pointed out above that the state $\rho \in W(0, T)$ solves the linear heat equation (A.1) with a right-hand side $\hat{f} \in L^{2}(Q)$. Depending on the particular Assumption 6.9-(i) on $c$, the function $\hat{f}$ is given by (A.2) or by (A.3). Now the claimed regularity follows from Lemma A.1.

Remark A.2. In addition to the continuous embedding (A.6), also the embedding

$$
W^{1,2}\left(S ; L^{2}(\Omega)\right) \cap L^{2}\left(S ; \operatorname{dom}_{L^{2}(\Omega)} \Delta\right) \hookrightarrow C^{1 / 2}\left(\bar{S} ; L^{2}(\Omega)\right)
$$

is continuous. Therefore, by interpolation arguments, $c f$. Triebel [37] the same holds for the embedding

$$
W^{1,2}\left(S ; L^{2}(\Omega)\right) \cap L^{2}\left(S ; \operatorname{dom}_{L^{2}(\Omega)} \Delta\right) \hookrightarrow C^{\alpha}\left(\bar{S} ; H^{1 / 2-\alpha}(\Omega)\right),
$$

with $0<\alpha<\frac{1}{2}$. In view of this, the embedding

$$
W^{1,2}\left(S ; L^{2}(\Omega)\right) \cap L^{2}\left(S ; \operatorname{dom}_{L^{2}(\Omega)} \Delta\right) \hookrightarrow C\left(\bar{S} ; L^{2}(\Omega)\right),
$$

is compact. Therefore, for the heat equation (A.1), the mapping $\hat{f} \mapsto \rho$ is compact from $L^{2}(Q)$ to $C\left([0, T] ; L^{2}(\Omega)\right)$.

Acknowledgements. We thank Joachim Rehberg (WIAS Berlin) for communicating to us the proof of Lemma 7.1. Moreover, we are grateful to Hannes Meinlschmidt (RICAM Linz) for his support in proving the statements of Remark 7.1 and to Eduardo Casas, who informed us about ([30], Thm. 4.1, p.153) after the paper had been accepted for publication. We also acknowledge the referees for their useful and detailed remarks that helped us to essentially improve the manuscript. 


\section{REFERENCES}

[1] G. Albi, Y.-P. Choi, M. Fornasier and D. Kalise, Mean field control hierarchy. Appl. Math. Optim. 76 (2017) $93-135$.

[2] G. Albi, L. Pareschi and M. Zanella, Boltzmann-type control of opinion consensus through leaders. Philos. Trans. R. Soc. Lond. Ser. A Math. Phys. Eng. Sci. 372 (2014) 20140138.

[3] H. Amann, Linear and quasilinear parabolic problems. Abstract linear theory. Vol. I, Vol. 89 of Monographs in Mathematics. Birkhäuser Boston, Inc., Boston, MA (1995).

[4] M. Annunziato and A. Borzì, Optimal control of probability density functions of stochastic processes. Math. Model. Anal. 15 (2010) 393-407.

[5] M. Annunziato and A. Borzì, A Fokker-Planck control framework for multidimensional stochastic processes. J. Comput. Appl. Math. 237 (2013) 487-507.

[6] M. Annunziato and A. Borzì, A Fokker-Planck control framework for stochastic systems. EMS Surv. Math. Sci. 5 (2018) 65-98.

[7] M.S. Aronna, J.F. Bonnans, and A. Kröner, Optimal control of infinite dimensional bilinear systems: application to the heat and wave equations. Math. Program. 168 (2018) 717-757.

[8] M.S. Aronna and F. Tröltzsch, First and second order optimality conditions for the control of Fokker-Planck equations. Preprint (2020) arxiv.org/abs/2002.03988v1.

[9] M.S. Aronna and F. Tröltzsch, First and second order optimality conditions for the control of Fokker-Planck equations. Preprint (2021) arxiv.org/abs/2002.03988v3.

[10] A. Ashyralyev and P.E. Sobolevskiĭ, Well-posedness of parabolic difference equations. Vol. 69 of Operator Theory: Advances and Applications. Translated from the Russian by A. Iacob. Birkhäuser Verlag, Basel (1994).

[11] J.-P. Aubin, Un théorème de compacité. CR Acad. Sci. Paris 256 (1963) 5042-5044.

[12] T. Bose and S. Trimper, Stochastic model for tumor growth with immunization. Phys. Rev. E 79 (2009) 051903.

[13] T. Breiten, K. Kunisch and L. Pfeiffer, Control strategies for the Fokker- Planck equation. ESAIM: COCV 24 (2018) $741-763$.

[14] T. Breiten, K. Kunisch and L. Pfeiffer, Infinite-horizon bilinear optimal control problems: sensitivity analysis and polynomial feedback laws. SIAM J. Control Optim. 56 (2018) 3184-3214.

[15] P. Cardaliaguet, Notes on mean field games. Technical report, 2010.

[16] E. Casas and F. Tröltzsch, Second order analysis for optimal control problems: Improving results expected from abstract theory. SIAM J. Optim. 22 (2012) 261-279.

[17] E. Casas, D. Wachsmuth and G. Wachsmuth, Second-order analysis and numerical approximation for bang-bang bilinear control problems. SIAM J. Control Optim. 56 (2018) 4203-4227.

[18] P.-H. Chavanis, Nonlinear mean field Fokker-Planck equations. application to the chemotaxis of biological populations. Eur. Phys. J. B 62 (2008) 179-208.

[19] M. Chipot, Elements of nonlinear analysis. Birkhäuser (2012).

[20] R. Dautray and J.-L. Lions, Mathematical analysis and numerical methods for science and technology: Evolution Problems I, volume 5. Springer Science \& Business Media (1992).

[21] R. Duan, M. Fornasier and G. Toscani, A kinetic flocking model with diffusion. Commun. Math. Phys. 300 (2010) 95-145.

[22] L.C. Evans, Partial differential equations. American Mathematical Society (2010).

[23] A. Fleig and R. Guglielmi, Optimal control of the Fokker-Planck equation with space-dependent controls. J. Optim. Theory Appl. 174 (2017) 408-427.

[24] G. Furioli, A. Pulvirenti, E. Terraneo and G. Toscani, Fokker-Planck equations in the modeling of socio-economic phenomena. Math. Models Methods Appl. Sci. 27 (2017) 115-158.

[25] V. Girault and P.-A. Raviart, Finite element methods for Navier-Stokes equations: theory and algorithms. In vol. 5. Springer Science \& Business Media (2012).

[26] D.A. Gomes and J. Saúde, Mean field games models - a brief survey. Dyn. Games Appl. 4 (2014) 110-154.

[27] M. Herty, C. Jörres and A.N. Sandjo, Optimization of a model Fokker-Planck equation. Kinet. Relat. Models 5 (2012).

[28] A.D. Ioffe, Necessary and sufficient conditions for a local minimum 3: Second order conditions and augmented duality. SIAM J. Control Optim. 17 (1979) 266-288.

[29] T. Kato, Perturbation theory for linear operators, Reprint of the corr. print of the 2nd edition. Classics in Mathematics. Springer-Verlag New York, Inc., New York (1980).

[30] O.A. Ladyženskaja, V.A. Solonnikov and N.N. Ural'ceva, Linear and quasilinear equations of parabolic type. Translated from the Russian by S. Smith. Translations of Mathematical Monographs, Vol. 23. American Mathematical Society, Providence, R.I. (1968).

[31] J.-M. Lasry and P.-L. Lions, Mean field games. Jpn. J. Math. 2 (2007) 229-260.

[32] J.-L. Lions and E. Magenes, Problèmes aux limites non homogènes et applications. Vol. 1. Dunod, Paris (1968).

[33] E.M. Ouhabaz, Analysis of heat equations on domains. Vol. 31 of London Mathematical Society Monographs Series. Princeton University Press, Princeton, NJ (2005).

[34] S. Roy, M. Annunziato and A. Borzì, A Fokker-Planck feedback control-constrained approach for modelling crowd motion. J. Comput. Theor. Transport 45 (2016) 442-458.

[35] L. Ryzhik, Lectures notes (on mean field games). Technical report (2018) available at: link.

[36] M. Schienbein and H. Gruler, Langevin equation, Fokker-Planck equation and cell migration. Bull. Math. Biol. 55 (1993) 585-608. 
[37] H. Triebel, Interpolation theory, function spaces, differential operators. Vol. 18 of North-Holland Mathematical Library. NorthHolland Publishing Co., Amsterdam-New York (1978).

[38] F. Tröltzsch, Optimal control of partial differential equations. Theory, methods and applications, Vol. 112 of Graduate Studies in Mathematics. American Mathematical Society, Providence, RI (2010). 